\title{
Fenêtres ouvertes sur le chemin du Midi : portraits des aires le long de l'Autoroute du Soleil, entre Beaune et Marseille
}

Looking out on the road to the South: portraying rest areas on the highway between Beaune and Marseille

Evocaciones de la ruta hacia el Sur de Francia: valoración paisajista de las áreas de servicio de la Autopista del Sol entre Beaune y Marsella

\section{Vincent Marchal}

\section{OpenEdition}

\section{Journals}

Édition électronique

URL : http://journals.openedition.org/mappemonde/276

DOI : 10.4000/mappemonde.276

ISSN : 1769-7298

Éditeur

UMR ESPACE

Référence électronique

Vincent Marchal, «Fenêtres ouvertes sur le chemin du Midi : portraits des aires le long de l'Autoroute du Soleil, entre Beaune et Marseille», Mappemonde [En ligne], 124 | 2018, mis en ligne le 10 juillet 2018, consulté le 20 juin 2019. URL : http://journals.openedition.org/mappemonde/276 ; DOI : 10.4000/ mappemonde. 276

Ce document a été généré automatiquement le 20 juin 2019. 


\title{
Fenêtres ouvertes sur le chemin du Midi : portraits des aires le long de l'Autoroute du Soleil, entre Beaune et Marseille
}

\author{
Looking out on the road to the South: portraying rest areas on the highway \\ between Beaune and Marseille \\ Evocaciones de la ruta hacia el Sur de Francia: valoración paisajista de las áreas \\ de servicio de la Autopista del Sol entre Beaune y Marsella
}

\section{Vincent Marchal}

«Il rentrait chez lui, là-haut, vers le brouillard,
elle descendait, là-bas, dans le Midi ». Michel
Fugain, Une belle histoire, 1972.

«Faites le plein de ressources sur nos aires ", répètent les panneaux à message variable installés sur le réseau autoroutier français, lors des grandes migrations de l'été. Au fil d'un trajet de vacances souvent rythmé par des contraintes horaires fortes (arrivéedépart des lieux de vacances), l'arrêt sur une aire obéit d'abord à des raisons utilitaires (restauration, passage aux sanitaires, carburant, achat en boutique) ou liées au confort physique (relaxation, marche, détente) ${ }^{1}$. À première vue, de nombreux usagers de ces aires les rattachent immédiatement à ce que certains scientifiques qualifient de "nonlieux " (Augé, 1992), qui peinent à se démarquer des territoires fonctionnels de l'automobile (Dupuy, 1995 ; Merriman, 2007). Mais, par-delà ces motivations d'ordre pratique, l'incitation à la halte est aussi l'occasion de sortir de l'habitacle du véhicule pour retrouver une expérience sensorielle totale, nouer une relation, même éphémère, avec les territoires traversés. Tiraillées entre l'exigence d'offrir des services standardisés, attendus, et le désir d'être fidèles à « l'esprit des lieux » traversés, les aires sont des objets géographiques paradoxaux. 
2 Sur la section la plus fréquentée de l'Autoroute du Soleil, le long d'un axe Saône-Rhône où «tout est transition» (Dubesset, 1972), l'horizon d'attente des vacances, la quête du dépaysement une fois quittés les territoires du quotidien, sont des motivations puissantes qui prêtent au paysage de cet axe un statut particulier dans la culture française, voire européenne. La succession des aires au fil de l'itinéraire amène à déterminer si ces dernières sont en mesure d'offrir au voyageur un aperçu significatif des territoires abordés, tout en restituant le passage entre les ambiances septentrionales et l'univers méditerranéen, trame de fond qui donne au trajet sa signification la plus forte aux yeux des nombreux estivants empruntant l'autoroute.

Le premier temps de la démarche part du ressenti des voyageurs sur les principales aires (d'après enquêtes), avec comme fil conducteur le positionnement du passage entre la France du Nord et celle du Midi. À partir de ces analyses, l'étude des aménagements des aires tentera de vérifier si ces derniers sont en adéquation avec les impressions collectées : si certains aménagements paysagers (espaces verts, architecture) expriment et justifient la transition dans toutes ses nuances, la mise en tourisme des aires tend à nier le principe même du passage progressif entre Nord et Midi.

\section{Entre lecture fonctionnelle de l'espace et promesse d'évasion : paroles d'automobilistes...}

4 Principaux clients, acteurs et consommateurs de l'autoroute, les automobilistes donnent aux aires leur raison d'être: si une écrasante majorité de vacanciers s'arrête pour des nécessités d'ordre pratique, il semble a priori difficile de s'écarter d'une lecture première de l'espace, à la fois fonctionnelle et marchande (IFOP, 2001), polarisée par la circulation (difficultés de stationner, nécessaire prudence lors des déplacements à pied), les pratiques d'achat en boutique (articles de plus en plus standardisés, dénués de références locales), et parfois le sentiment d'insécurité (vigilance face aux vols ou petits délits). Les enquêtes menées sur les aires ont tenté de dépasser cette approche utilitaire dominante de l'espace, en travaillant sur l'horizon d'attente spécifique à l'Autoroute du Soleil, fondé sur la quête des vacances et du dépaysement, alimentée par le passage de la France du Nord au Midi méditerranéen, clé de lecture fondamentale de la séquence paysagère entre Beaune et Marseille (Marchal, 2013). Sur les aires, la première difficulté fut de nouer un contact avec des automobilistes souvent pressés (horaires des locations...) et en famille, méfiants, voire agressifs, peu enclins à être dérangés. La nécessité d'établir une enquête courte, semi-guidée (Paulet, 2002) s'imposait afin de collecter un nombre significatif de témoignages. Deux séries d'enquêtes ont été menées à l'été $2013^{2}$.

\section{Parfum d'évasion, avant-goût de vacances : une alchimie spécifique aux aires de l'autoroute du Soleil ?}

5 Une première enquête recense les données qui nourrissent la sensation de « dépaysement " (Roger, 19973) partagée par de nombreux vacanciers arrêtés sur une aire, en cherchant à distinguer ce qui relève de l'ambiance interne de l'aire et les éléments qui constituent une trame de fond sur la séquence du trajet. La question «À cet endroit, vous sentez-vous déjà dépaysé ? Pour quelles raisons?» ne retenait pas les voyageurs plus d'une minute, tandis que leur provenance géographique était également 
notée. Sur la figure 1, les résultats ont été portés pour toutes les aires, en séparant celles de repos, plus petites, moins fréquentées, sur lesquelles l'échantillon de réponses a été limité à 50, et les aires de service, les plus prisées pour les commodités offertes, où il a été facile de totaliser à chaque fois 100 réponses.

Figure 1. Enquête consacrée à la sensation de dépaysement sur les aires de la section BeauneMarseille et traitement des réponses

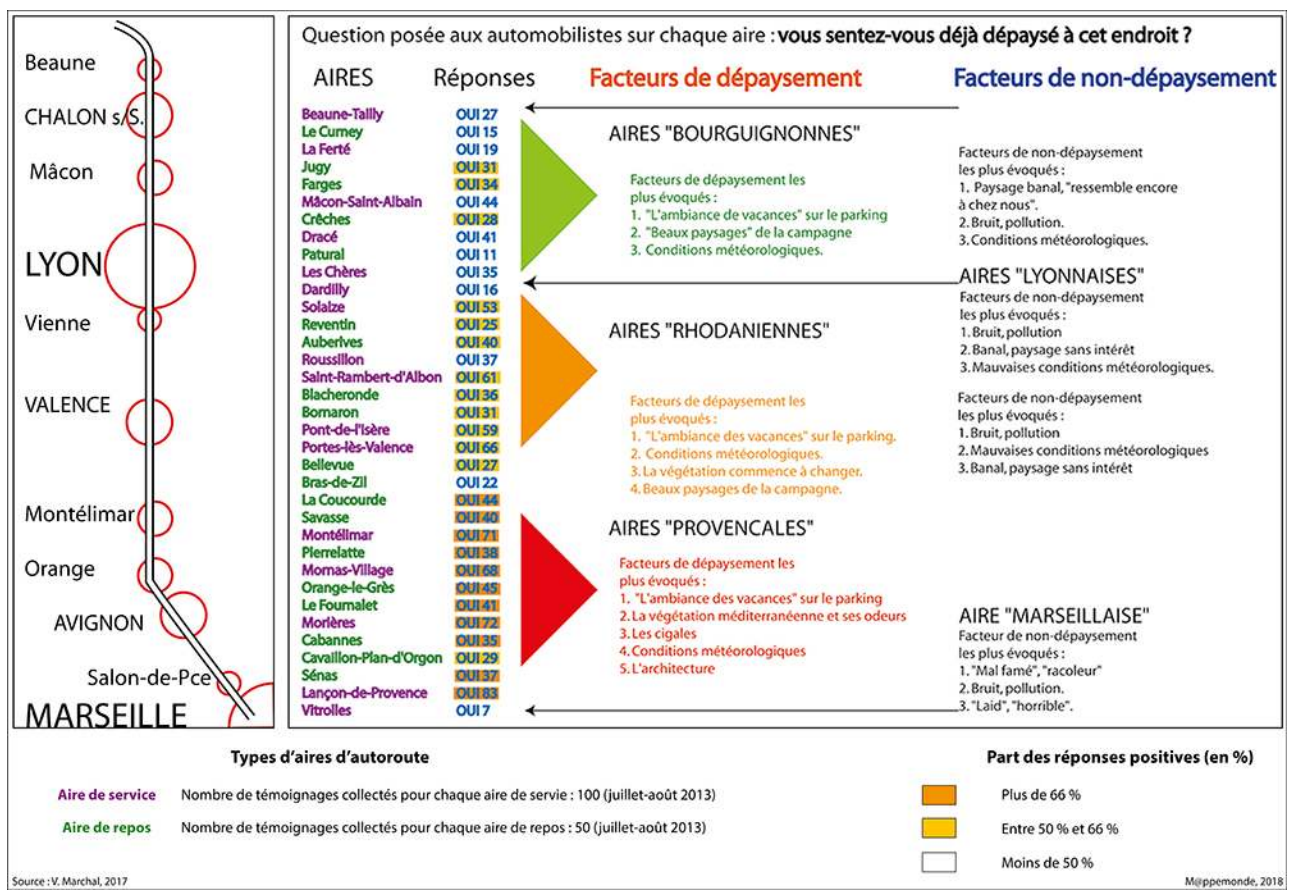

Source : V. Marchal, 2017

6 Les résultats sur l'ensemble du tronçon font ressortir plusieurs ensembles d'aires : au nord de Lyon, le sentiment de dépaysement n'est pas absent, mais il n'est pas encore général. Pour une majorité d'estivants gagnant le Midi méditerranéen, les aires bourguignonnes sont associées à des lieux intermédiaires, banalisés, dont le cadre paysager serait encore une périphérie de leur environnement quotidien: mosaïque agraire associant openfield et bocage, fondé sur la trilogie blé-maïs-élevage, cortège végétal des aires assez plastique, mésoeuropéen (photo 1). Rapidement associées à des espaces sans attrait particulier, les aires sont aussitôt décrites par ces usagers nondépaysés (originaires à $70 \%$ de la région parisienne, du nord et du nord-est) comme des espaces bruyants, peu propices à une évasion hors des territoires de l'automobile qui imposent aux lieux leur personnalité souveraine. Mais certaines haltes au bord de l'A6 alimentent un ressenti ambivalent, car d'autres y trouvent déjà ce que la société concessionnaire APRR nommait en 1995 un "avant goût de vacances, une singulière impression d'évasion $»^{4}$. Cependant, ce sentiment de dépaysement relève davantage des circonstances de l'arrêt (jour de départs, météo clémente), de la fréquentation de l'aire par d'autres estivants en partance («atmosphère de vacances», figure 1) que de l'agencement interne des aires et de leur paysage environnant. Ce dernier n'est évoqué qu'en second lieu, uniquement en présence d'un temps dégagé et chaud: lorsqu'il est investi d'une charge positive, le paysage est rarement celui de l'aire, mais qualifie plutôt les échappées visuelles au-delà des grillages. Cette sensation n'est d'ailleurs relevée que sur les aires de repos (absence de stations et boutiques, vues comme des «anti- 
paysages ») où la végétation discontinue garantit des échappées visuelles sur l'extérieur (Jugy, Farges photo 2, Crêches). Les automobilistes qui trouvent dans les campagnes de «la verdoyante Bourgogne » (Dunlop et Cortazar, 1983) une première marque d'évasion entrent dans le mécanisme des sociétés postmodernes, valorisant une campagne appréciée sous le signe de l'authentique, du singulier, de la «nature » (paysage bocager), tandis que l'urbain reste associé au domaine de la modernité et de la standardisation.

Photo 1. L'aire de Crêches s'ouvre sur un paysage agricole banal, « un grand champ de blé » digne des amoureux de M. Fugain

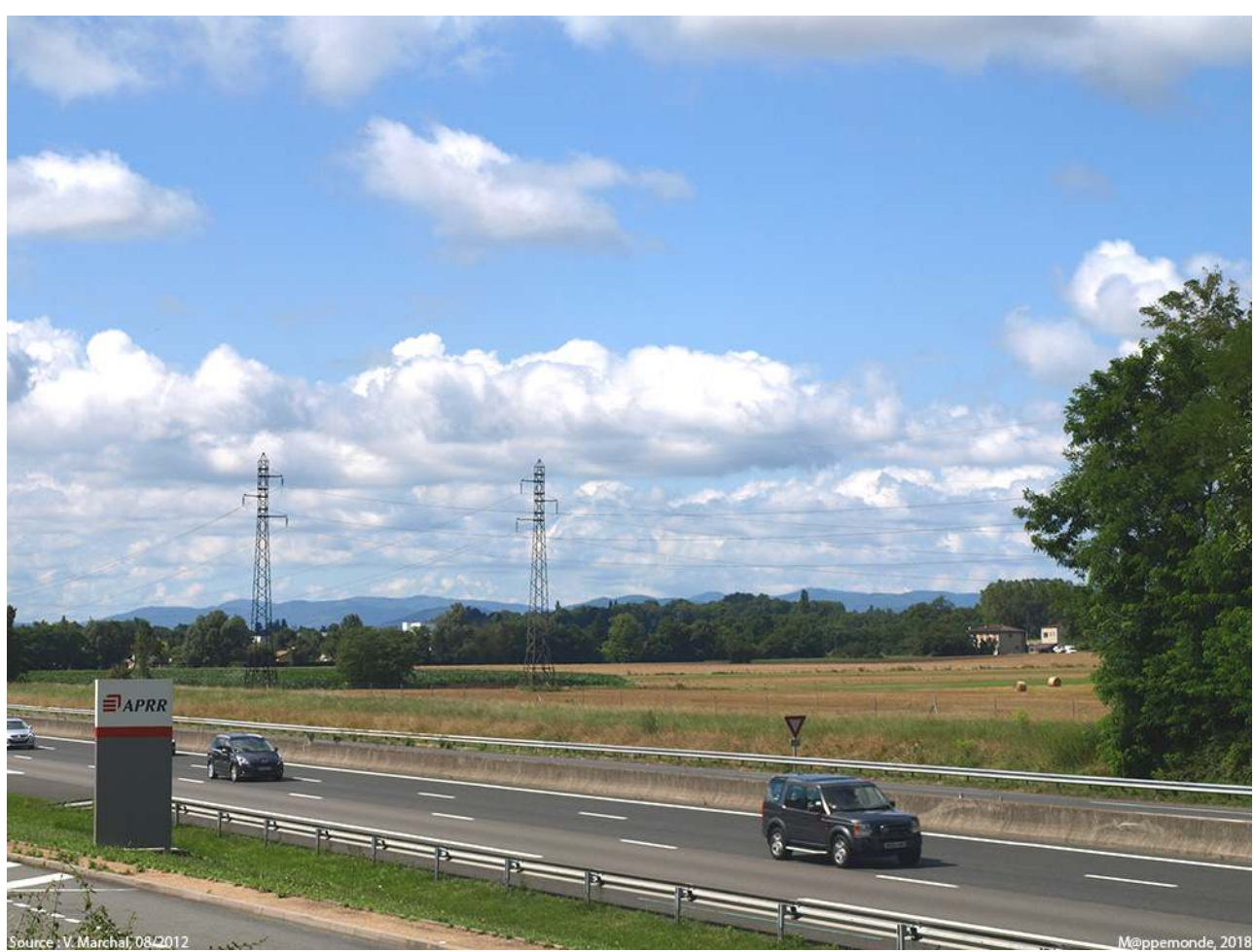

Cliché V.Marchal., 08/2012

Photo 2. L'aire de Farges permet de découvrir le village du même nom, avec ses habitations en pierre calcaire, et les premières parcelles du vignoble mâconnais

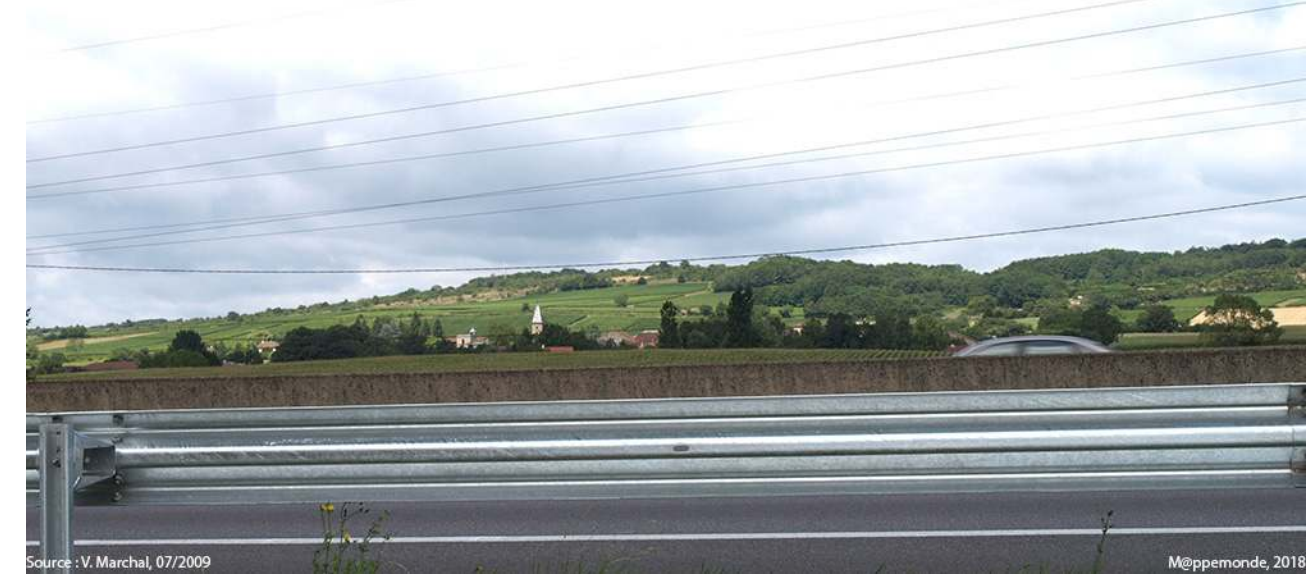

Cliché V. Marchal., 07/2009 
Ce rapport dual aux identités urbaines et rurales culmine sur la section gratuite de l'autoroute du Soleil dans sa traversée de Lyon. Minuscule, " sale », " moche ", « à gerber [sic] » l'aire de Dardilly à $5 \mathrm{~km}$ du centre, est perçue par les voyageurs en transit comme une marge fonctionnelle au cœur de la métropole rhônalpine, sorte de non-paysage, dépourvu de tout ancrage territorial. En revanche, l'aire de Solaize, accolée au " couloir de la chimie ", est investie d'une nouvelle sensation de dépaysement, qui doit davantage à la situation de l'arrêt qu'à son aménagement (sortie de la vallée de chimie, de la métropole lyonnaise et de ses difficultés de circulation éventuelles, regroupées par les automobilistes dans un vaste « complexe lyonnais » porteur d'images négatives).

Passé l'épisode urbain lyonnais, l'impression de dépaysement s'accroît tout au long de l'A7. Toutefois, les enquêtes amènent à distinguer deux ensembles d'aires (figure 1). Les haltes "rhodaniennes » (section Vienne-Valence) trouvent aux yeux des voyageurs une image positive, car elles sont le signe d'une atmosphère rurale retrouvée, sur laquelle l'immersion dans le monde méridional sera plus facile à lire que dans un espace urbain saturé en références. Si le contexte de l'ambiance des départs propre à toute l'autoroute à ce moment de l'année reste encore évoqué en premier, les changements végétaux et climatiques remarqués avant l'arrêt, puis sur l'aire, font désormais du dépaysement une expérience sensorielle totale (essences et odeurs nouvelles [photo 3], chaleur, bouffées de Mistral, parfois, chant des cigales, sont spontanément évoqués par les automobilistes). Cependant, les variations météorologiques restent le critère qui arbitre de nombreux ressentis, puisque, les jours de météo mauvaise (pluie, vent, temps frais et couvert), la part des usagers dépaysés tombe à moins de $30 \%$, quel que soit l'environnement paysager (photo 4).

Photo 3. Les collines du Bas-Dauphiné, vues depuis l'aire de Saint-Rambert-d'Albon

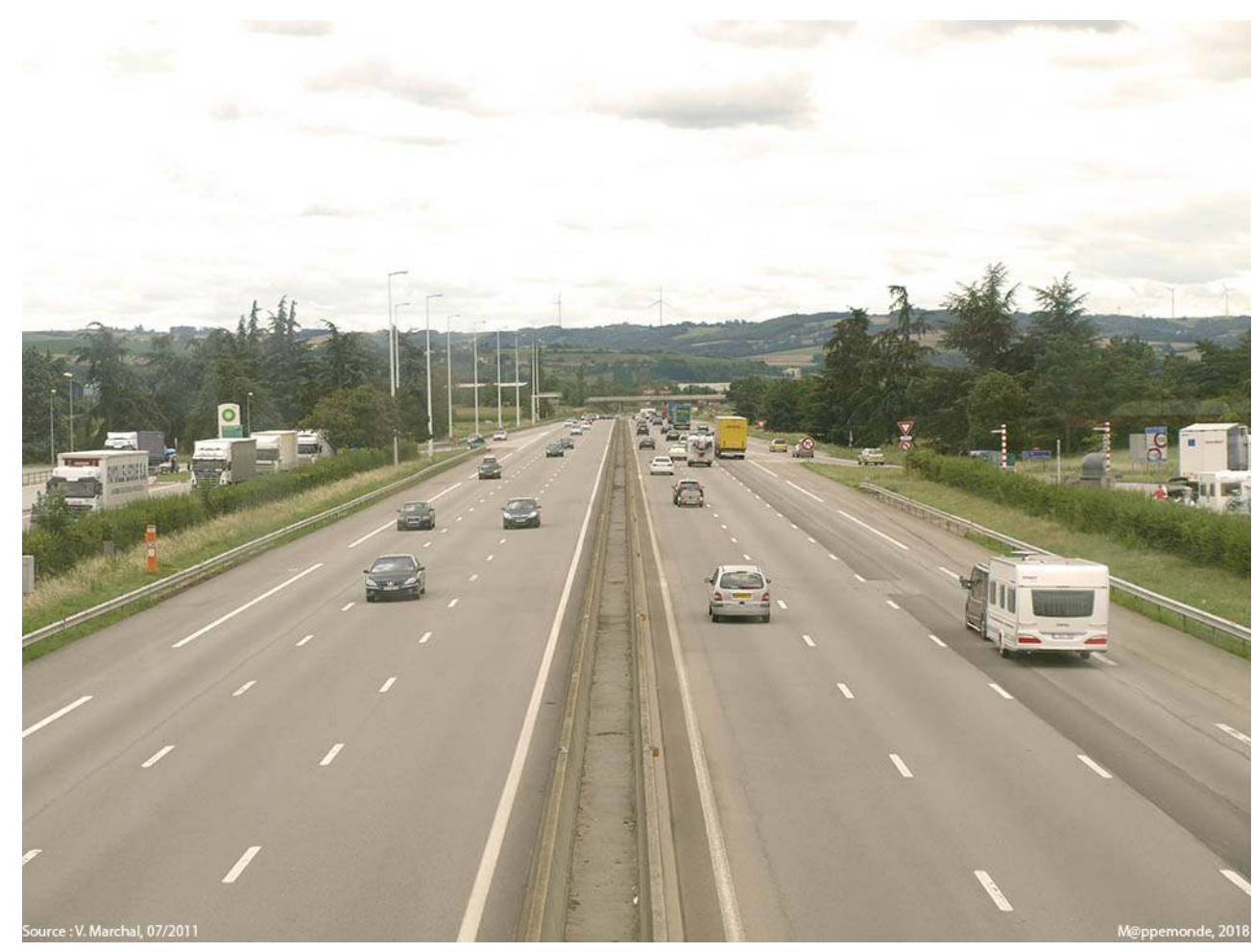

L'humeur météorologique du jour donnera ou non à ce paysage les premiers accents méridionaux. Cliché V. Marchal, 07/2011 
Photo 4. Aire de Saint-Rambert-d'Albon

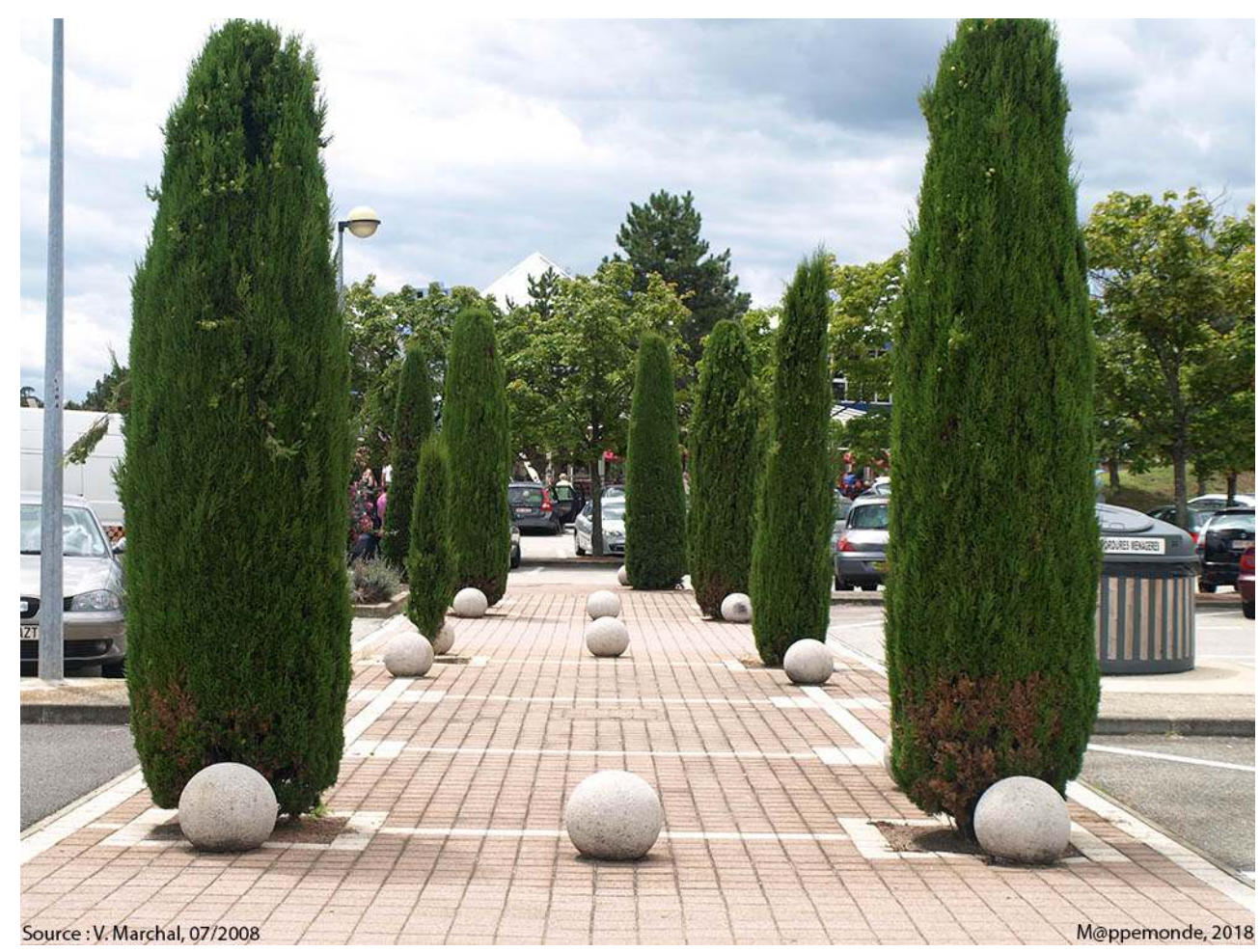

Promenade au milieu des cyprès et des mûriers, taquinés par le Mistral.

Cliché V. Marchal, 07/2008

9 Avec l'entrée dans la plaine de Montélimar, le dépaysement devient réalité sur toutes les aires de la section, en raison de l'immersion dans l'univers méditerranéen, qui rend imminente l'approche du but pour de nombreux vacanciers, qui n'hésitent pas à parler d'aires «provençales». Variables essentielles de l'expérience sensorielle à la sortie du véhicule, les données météorologiques ne sont plus déterminantes pour assurer le dépaysement, bien qu'elles le confortent: même les jours de temps couvert, avec des températures refroidies par le Mistral $\left(20^{\circ} \mathrm{C}\right)$, le cortège végétal méditerranéen donne sa personnalité aux lieux, et la sécheresse estivale insinue ses marques sur l'ensemble du paysage (garrigue, pins, pelouse brûlée par le soleil: photo 5), à tel point que le dépaysement s'impose comme une évidence. 


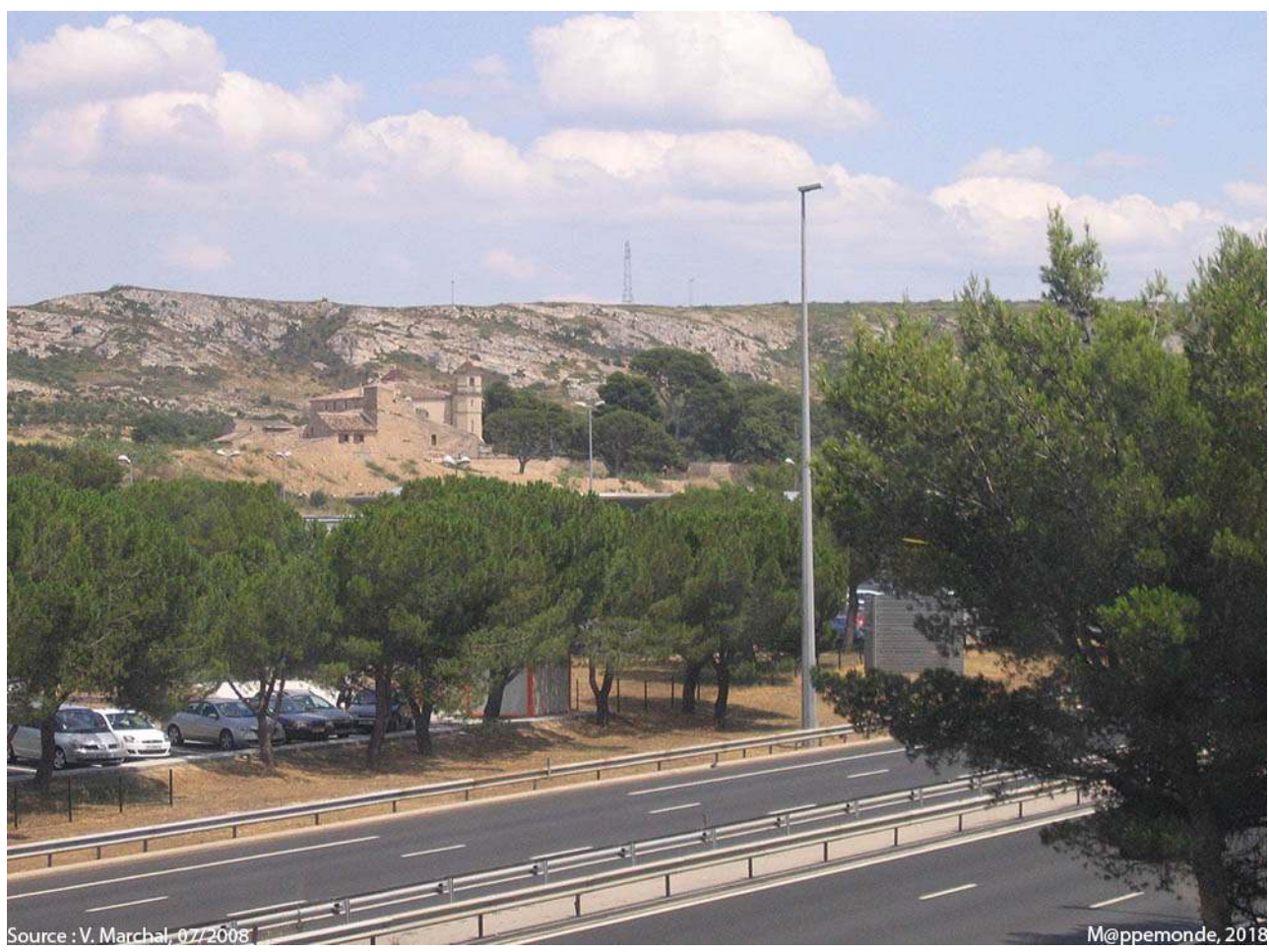

Depuis le pont- restaurant de Lançon, vue sur le Mas Sénéguier dans un écrin de garrigue. Cliché V. Marchal, 07/2008

\section{La transition entre France du nord et du Midi, trame de fond du dépaysement}

Partant du constat que le dépaysement sur l'autoroute du Soleil était intimement lié à la transition entre la France du «Nord» et celle du «Midi», une seconde enquête a été réalisée sur les aires. Dans la mesure où les notions de " Midi », " monde méditerranéen », «Provence » étaient synonymes pour beaucoup d'automobilistes, le choix des termes à retenir n'allait pas de soi. La Provence renvoie à une réalité historique, administrative et culturelle qui s'inscrit en grande majorité dans l'univers méditerranéen, qu'elle tend parfois à résumer, par synecdoque. De même, restreindre le questionnement au Midi méditerranéen risquait d'occulter la richesse des représentations associées au Midi, qui se décline au pluriel (ainsi, certains usagers affirmaient que Valence était dans le Midi, mais pas encore méditerranéenne). Conserver le terme générique permettait de faire du Midi une catégorie d'espace, un « champ spatial » à géométrie variable investi de définitions tantôt larges (le « Sud»), tantôt restreintes (« Midi méditerranéen »)

La question « vous sentez-vous dans le Midi à cet endroit ? Pourquoi ? » a été soumise aux vacanciers selon les mêmes modalités que la première enquête (figure 2). À l'analyse, très peu d'automobilistes se sentent dans le Midi au nord de Lyon, les seules réponses affirmatives étant celles de voyageurs étrangers, venus d'Allemagne, des Pays-Bas et du Royaume-Uni, pour lesquels le Midi est plutôt synonyme d'un « ailleurs » plus ensoleillé. 
Figure 2. Enquête consacrée au seuil du Midi sur les aires de la section Beaune-Marseille et traitement des réponses

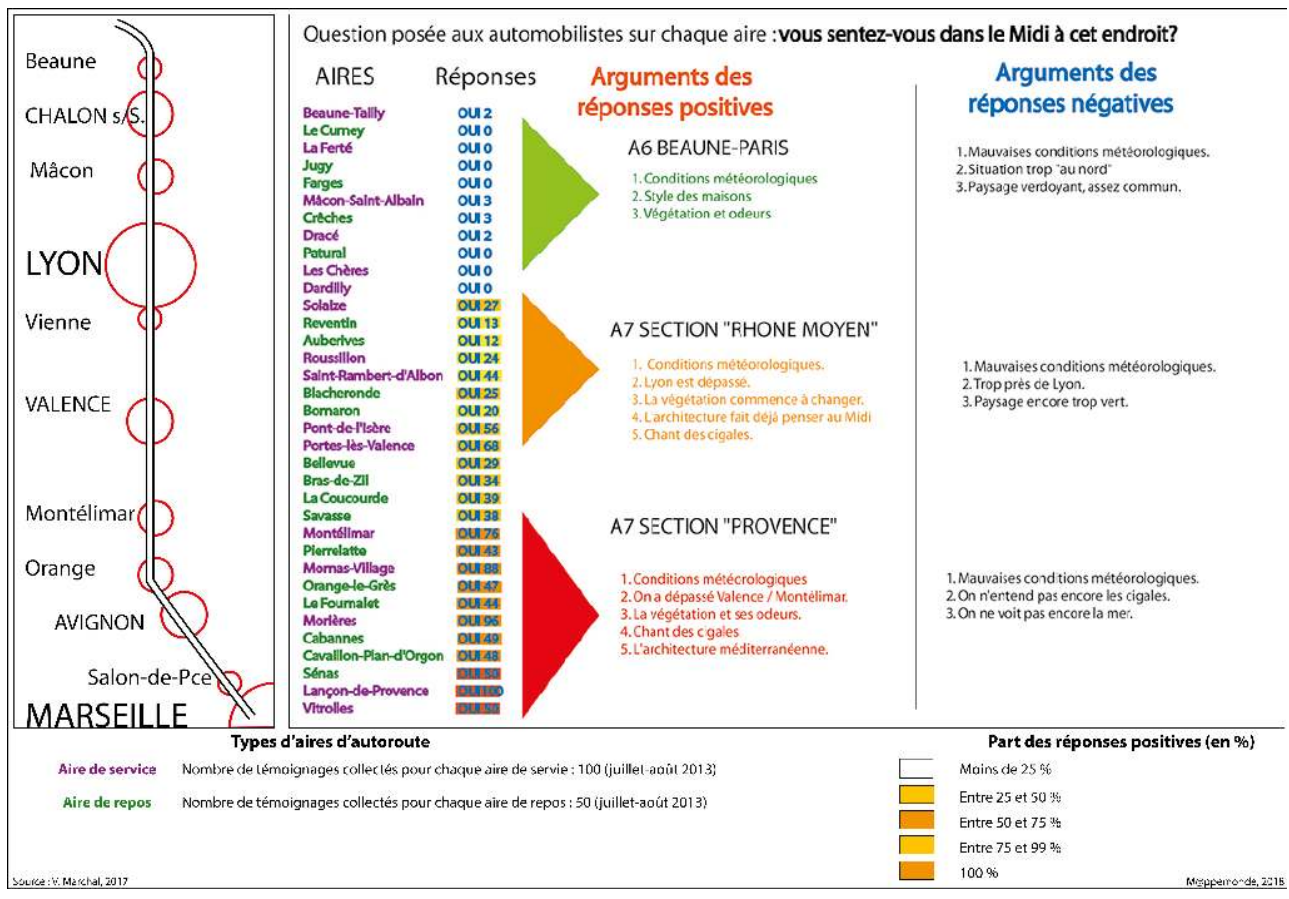

Source : V. Marchal, 2017

12 La confrontation des deux enquêtes indique qu'au sud de Lyon, les seuils du Midi interfèrent avec ceux du dépaysement, notion qui renvoyait à un changement plus général. De nouveau, la météorologie commande fortement l'inscription de l'aire dans le monde méridional : de Lyon à Montélimar, les haltes rhodaniennes s'inscrivent dans une zone d'hésitation paramétrée par la température extérieure, les précipitations, plus encore que par le traitement végétal et architectural des lieux. Sur cette section, l'argument de la situation du lieu l'emporte sur la caractérisation du paysage, au final assez peu évoqué. La majorité des personnes interrogées sur ce tronçon mobilise d'abord des repères géographiques confortant leur délimitation a priori du Midi, et puise ensuite des arguments dans le cadre paysager environnant (végétation résineuse et ses effluves, style des constructions). Les témoignages recueillis prêtent à Montélimar la force d'un repère commun, audelà duquel s'ouvre le Midi «méditerranéen ». Une fois développé l'argument de la situation du lieu, les allusions aux ambiances végétales, olfactives, au ressenti de la température et au chant des cigales prouvent à quel point la plupart des automobilistes trouvent sur les aires "provençales » la vérification d'une définition du Midi à la fois zonale et sensorielle.

13 Cependant, malgré l'insistance sur l'analyse du paysage " à cet endroit ", une des limites des enquêtes fut la difficulté à séparer dans le discours ce qui relève de l'aire proprement dite et les paysages aperçus en amont, qui ont forgé le vécu spatial récent de l'individu prenant sa pause. Par-delà ce flou difficile à clarifier dans les réponses, il apparaît que le traitement végétal des aires est le paramètre d'aménagement le plus puissant pour anticiper le « dépaysement » des vacances. 


\section{Le décor végétal : des portraits tout en nuances et en transition?}

14 L'immersion dans un cadre nouveau est suggérée par les ambiances végétales, qui campent une toile de fond, une suite de «décors» (Boutefeu, 2007) orchestrés différemment au fil des sections autoroutières. Les paroles d'automobilistes recueillies auparavant amenaient à distinguer l'autoroute A6, au fil de laquelle le paysage rural fixait la trame du dépaysement.

\section{Sur l'autoroute A6 : évasions et parties de campagne...}

Tournier à Dunlop et Cortazar, les romanciers prenant l'A6 pour décor ont décrit ce sentiment paradoxal de liberté qui commence au-delà du péage signifiant la sortie de la métropole francilienne. À défaut d'annoncer un Midi en décalage avec l'identité locale, APRR a très tôt saisi que pour les milliers de Parisiens prenant l'A6 au départ de la capitale, la sensation agréable de dépaysement naissait de l'authenticité d'une "nature " retrouvée, dans un cadre champêtre et verdoyant qui serait l'antithèse de la ville minérale décriée par la société postmoderne. Pour les vacanciers extérieurs aux régions traversées par l'infrastructure, l'entrée sur l'autoroute du Soleil signe la sortie du « temps économique et social quotidien » (Hägerstrand, 1975'), amplifiée par la sensation de parcourir un paysage "extra-ordinaire » (Lassus et al., 1994), scénarisé par le champ visuel très spécifique, la signalisation, les choix végétaux et architecturaux qui sont le fruit de la seule société exploitante du réseau. En ce sens, le paysage des aires d'autoroute investit le registre symbolique, celui de la "re-création ", en faisant du lieu à la fois « un signe et une signature » (Donadieu, Périgord, 2005) de l'espace abordé.

La figure 3 montre que la succession des aires de l'A6 esquisse plusieurs sous-catégories d'ambiances végétales (figure 3-2), qui restituent assez fidèlement les nuances de leur environnement biogéographique (figure 3-1) : aires très boisées entre Beaune et Chalon, elles-mêmes situées au cœur de vastes domaines forestiers (Bois de Servotte, forêt domaniale de Chagny), aires de plaine entre Chalon et Mâcon, agrémentées de peupliers, et ponctuées d'essences humifères faisant écho à l'atmosphère particulière du val de Saône, frangé de peupliers blancs et de ripisylves. En 1988, l'aire de Jugy, réaménagée sur le thème d'une promenade mycologique (photo 6), devait être un clin d'œil à l'identité très forestière de la Bourgogne, afin de combattre l'image d'une aire impersonnelle, en plein soleil (Dunlop, Cortazar, 1983). Néanmoins, entre Mâcon et Villefranche, les essences retenues sur les aires expriment un appel méridional en discordance avec les boisements environnants : sur l'aire de Crêches, "perdue au milieu des herbages et des marais de la plaine de la Saône ${ }^{7}$ » le voyageur peut être surpris de se trouver entouré de pins (figure 1). Ces choix s'expliquent, car l'A6 représente l'extension la plus méridionale du réseau APRR, et la société concessionnaire tient sans doute à créer à l'extrémité de son «autoroute du Soleil» l'avant-goût de vacances qui s'incarne dans une végétation à affinités méditerranéenne. 
Figure 3. Le traitement végétal des aires de la section Beaune-Lyon de l'autoroute A6 et sa perception par l'automobiliste

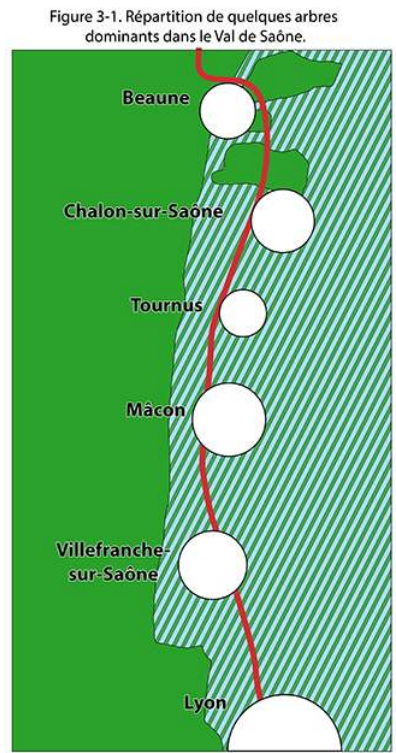

Futaie de hêtres, chênes et charmes

Essences humifères: frènes, ormes, aulnes, saules et bouleaux

IIII Peuplier : linéaires ou présence poncutelle

IIV. Pins sylvestres, cèdres, résineux étrangers au Source: V. Marchal, 2017
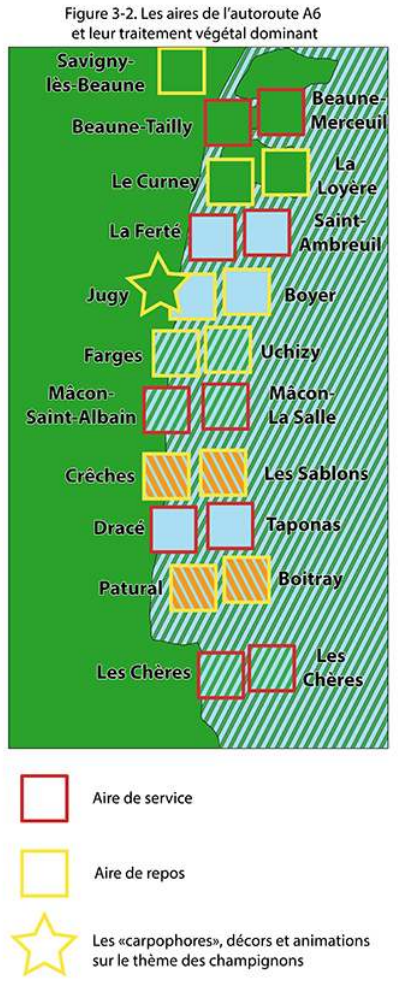

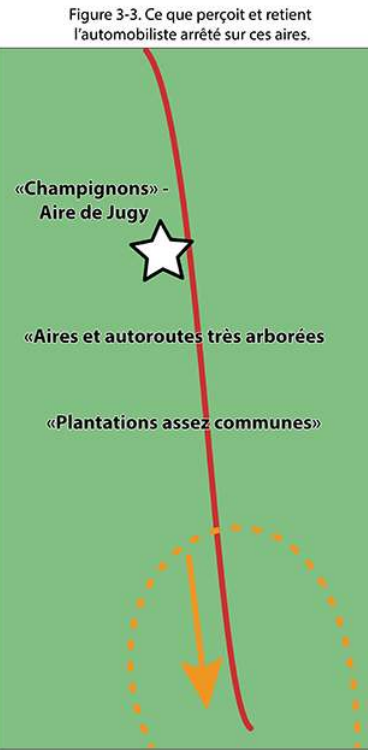

7 Réalisation remarquée par les usagers,

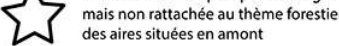
- - - Ambiance végétale «déja un peu du Midix

Photo 6. Promenade mycologique dans une Bourgogne encore très forestière

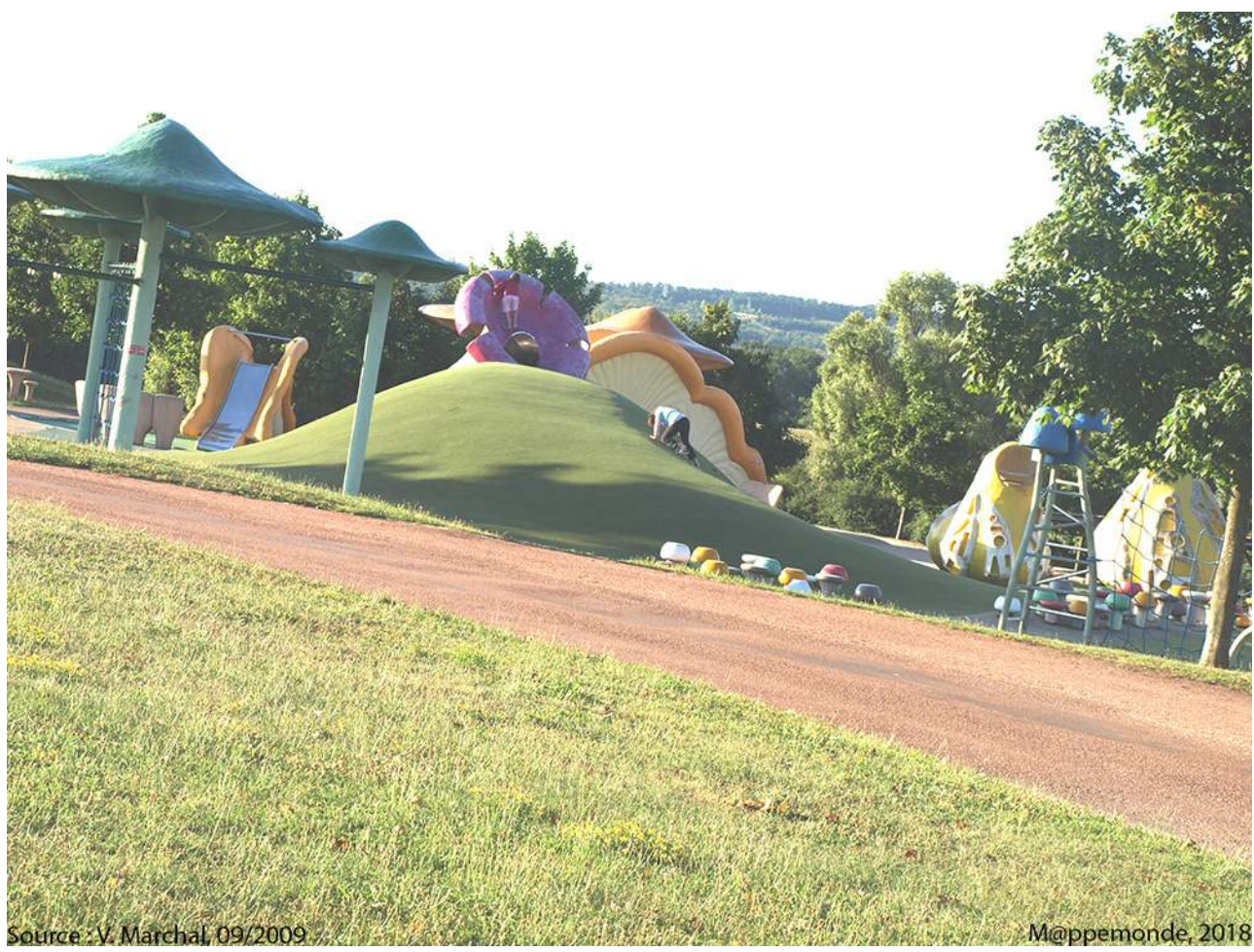

Aire de Jugy

Cliché V. Marchal, 09/2009 
Mais sur l'A6, cette volonté de fondre les aires dans leur cadre végétal environnant n'est pas remarquée par les automobilistes, au final peu attentifs à des plantations qu'ils jugent assez communes (figure 3-3), peut- être parce qu'elles se démarquent peu d'un environnement lui-même relativement ordinaire (futaies, campagne semi-bocagère du val de Saône). Les aménagements des aires, tout comme leur accompagnement végétal, sont ramenés à une sorte de paysage générique, une "espèce d'espace ». Toutefois, le faible intérêt culturel que prêtent les voyageurs à cet environnement n'exclut pas la sensation positive d'une autoroute verte, arborée, propice à la détente grâce à l'ombre généreuse dispensée par des arbres à maturité. Cette vision est déjà fort différente des verdicts dépréciatifs portés par les écrivains Dunlop et Cortazar, qualifiant d'« horreur " (Jugy), «rehorreur» (Farges), "autre horreur dans cet endroit où les horreurs abondent " (Crêches), des aires où la végétation encore jeune rendait l'ombre rare et précieuse.

Seuls les résineux au sud du réseau retiennent un peu l'attention des vacanciers à l'arrêt entre Mâcon et Villefranche, car leur port, leurs effluves, sont rattachés à l'annonce du Midi approchant.

\section{Les aires de l'autoroute A7 : « on dirait le Sud... »}

Sur la section payante de l'A7 (Vienne-Lançon, $300 \mathrm{~km}$ ), la transition bioclimatique prend son expression la plus éloquente, avec de nombreuses combinaisons végétales intermédiaires. Sur les aires comme sur le linéaire, la variété des motifs végétaux répond au désir précoce de bien intégrer l'infrastructure à son environnement paysager (rapport ASF, 1984). Mais au fil de l'A7, la diversité des ambiances paysagères et des attentes (surprendre l'approche du Midi) offre une occasion privilégiée de combattre le sentiment de monotonie encore souvent décrié par les usagers des autoroutes (Jardin et Fleury, 1973 ; IFOP, 2001). À la différence de l'A6, la société concessionnaire de l'autoroute tient cette fois à dévoiler la transition en respectant sa progressivité, avec ses nombreuses nuances intermédiaires. La figure 4-1 révèle la complexité de la flore rhodanienne, où se succèdent des sous-unités végétales cloisonnées par les reliefs d'orientation est-ouest (Lenoble, 1921; Garraud, 2005; Marchal, 2013), très sensibles aux différences d'exposition, bien visibles pour les vacanciers circulant sur l'autoroute (versants exposés plein sud et porteurs d'une flore plus méridionale). La confrontation des figures 4-1 et 4-2 exprime une certaine adéquation entre la végétation locale et celle des aires. Sur la section payante, la première véritable aire de repos (Auberives), très arborée et ombragée, est en connivence avec la ripisylve de la Varèze (peupliers, aulnes glutineux, acacias). Ce décor très verdoyant dépayse les estivants ( $80 \%$, figure 1 ) qui y trouvent des conditions propices à la détente après la traversée de Lyon, mais il n'est pas à proprement parler méridional (figure 2, $24 \%$ des usagers se sentent dans le Midi à cet endroit). Singulier " retour en arrière », comme s'il était nécessaire, passée la métropole lyonnaise, de réaffirmer le stéréotype de la «nature » synonyme de dépaysement et de liberté, et de soigner la première aire de la section exploitée par Vinci. Le traitement des aires suivantes se calque d'assez près sur les bassins végétaux intermédiaires qui s'enchaînent jusqu'à la Méditerranée : le mûrier faux-platane et le peuplier, taquinés par le Mistral, répondent au décor des plaines rhodaniennes (Saint-Rambert-d'Albon photo 4, Valence, Montélimar, Pierrelatte), le chêne pubescent et le robinier faux-acacia habillent les aires

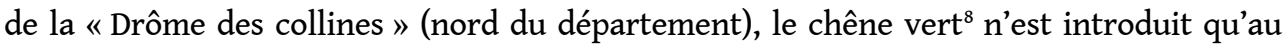
sud de Montélimar, au niveau des collines de Donzère, qui correspondent à la limite de 
son aire de répartition (Lenoble, 1921; Garraud, 2005). À partir de l'aire de Montélimar, les résineux prennent peu à peu le pas sur les feuillus (pins d'Alep, cèdres), à l'image de la flore des collines environnantes.

L'ensemble de ces essences est d'abord créateur d'ambiances, de décors (Boutefeu, 2007) sur lesquels se détachent des espèces au pouvoir d'évocation bien plus puissant aux yeux des automobilistes. Ainsi, le cyprès s'impose à partir des aires drômoises, et prend presque une fonction signalétique balisant le Midi. Au sud d'Orange, l'olivier est investi d'une charge symbolique encore plus forte, comme une "synecdoque paysagère" (Bigando, 2006) où s'incarne la Provence.

D'une aire à l'autre, il y a bien transition, dans la mesure où le changement d'atmosphère n'est jamais radical: l'apparition d'une espèce nouvelle est toujours accompagnée des essences déjà implantées sur les aires en amont et caractéristiques d'un plus vaste périmètre. À l'image de ce qui se produit dans tout le couloir rhodanien, la végétation des aires illustre le principe des ensembles flous, où interfèrent des systèmes parasites extérieurs à l'espace local (Rolland-May, 1987): l'apparition du cyprès en plaine de Valence (aire de Pont-de-l'Isère) a lieu sur fond de cèdres et d'acacias, qui s'étaient invités sur les aires en amont.

Critère d'appréciation essentiel lors de fortes chaleurs, le degré d'insolation des aires esquisse aussi un rythme spatial singulier (figure 4-3), qui conditionne le ressenti des usagers (figure 4-4). De Lyon à Marseille, les aires de repos de l'A7 entretiennent un rapport paradoxal avec le soleil, à l'image des civilisations méditerranéennes. Comme un but ultime de l'autoroute qui porte son nom, le soleil nourrit une identité positive lorsqu'il devient le support d'une mise en tourisme des territoires du sud de la France. Il cristallise l'imaginaire des vacanciers venus chercher sur la côte les «4S » (Knafou et Stock, 2008 $)$. Toutefois, si l'image du soleil est porteuse de rêve, son expérience immédiate sur les aires d'autoroute tend à ôter le plaisir lié à la halte si l'ombre n'est pas garantie. En d'autres termes, le contexte du voyage sur autoroute, qui suppose l'absence des « autres S ", ainsi que la fatigue cumulée du trajet déconnectent l'exposition au soleil des autres sources de plaisir qui lui sont associées. De Vienne à Pierrelatte, les haltes en plaine sont les moins ombragées, tandis que les aires étagées, dans un environnement collinaire (nord de la Drôme, reliefs calcaires au nord de Montélimar) garantissent une ombre plus généreuse (chênes pubescents, robiniers qui répondent aux forêts encadrant les aires). À partir de la Provence, la quête de l'ombre devient un précieux gage de qualité (places de stationnement, zone de pique-nique), dispensé par les vastes surfaces de recouvrement des pins (Amat in Le Cœur [dir.], 2002). Mais cette distinction entre aires ombragées et excessivement ensoleillées s'est opérée au fil des années, lorsque les écrans végétaux sont parvenus à maturité. En mai 1982, Dunlop et Cortazar faisaient encore état d'aires très découvertes, exposées au vent et au soleil, à l'exception des aires d'Auberives et de la Coucourde.

Sur la figure 4-4, le discours des automobilistes sur les aires illustre une corrélation forte entre la végétation et les ressentis climatiques : la sensation de chaleur est plus accusée sur les aires de plaine peu ombragées, exposées à un Mistral indissociable du Midi. Au fil de l'itinéraire, la température, le chant des cigales (plus intense sur les aires plantées de résineux) deviennent des marqueurs méditerranéens si puissants qu'ils finissent par éclipser les ambiances végétales dans les propos des estivants (aucun automobiliste n'est sensible à la végétation plus humifère des aires du val de Durance, entre Avignon et Sénas). De même, le fait d'avoir franchi certains points symboliques du trajet constitue 
aussi un paramètre de délimitation du Midi, indépendamment de l'ambiance instaurée sur les aires.

Figures 4-1, 4-2, 4-3. Le traitement végétal des aires de l'autoroute A7 et sa perception par l'automobiliste

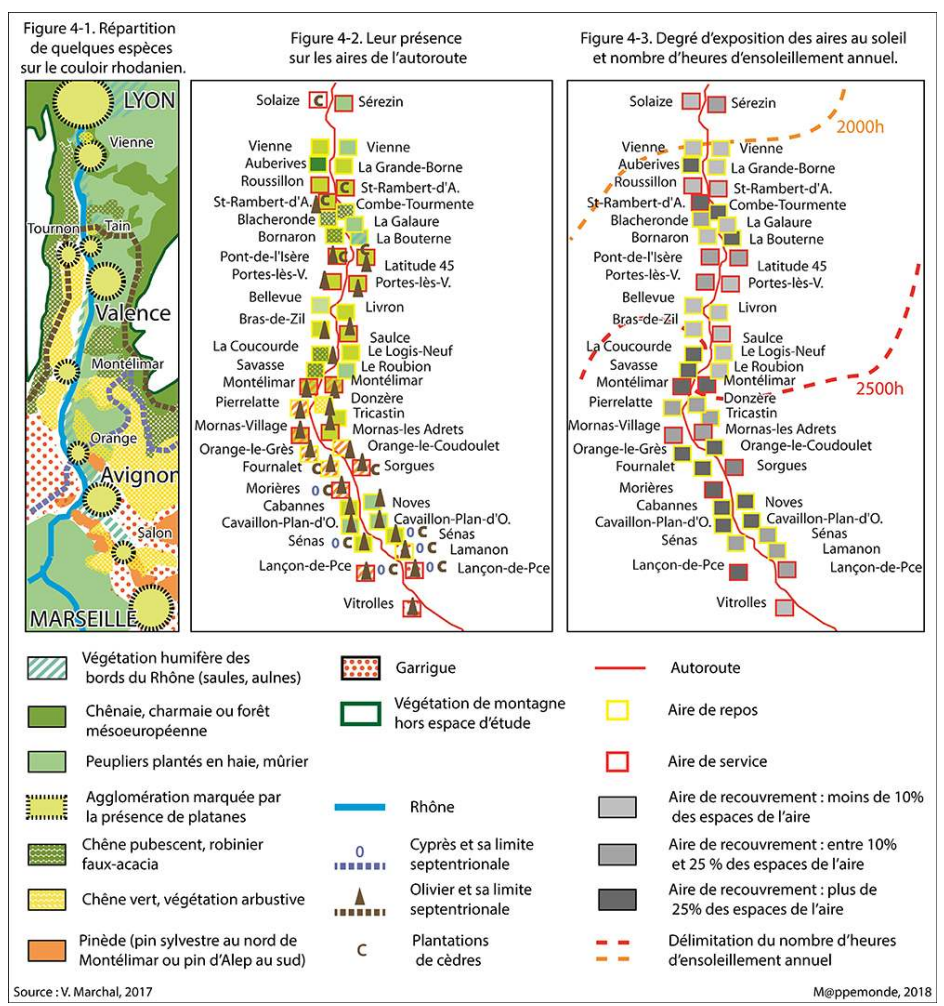

Figure 4-1. Répartition de quelques espèces sur le couloir Rhodanien Fique 4-2. Leur présence sur les aires de l'autoroute

Figure 4-3. Degré d'exposition des aires au soleil et nombre d'heures d'ensoleillement annuel Source : V. Marchal, 2017 
Figure 4-4. Ce que perçoit et retient l'automobiliste arrêté sur les aires

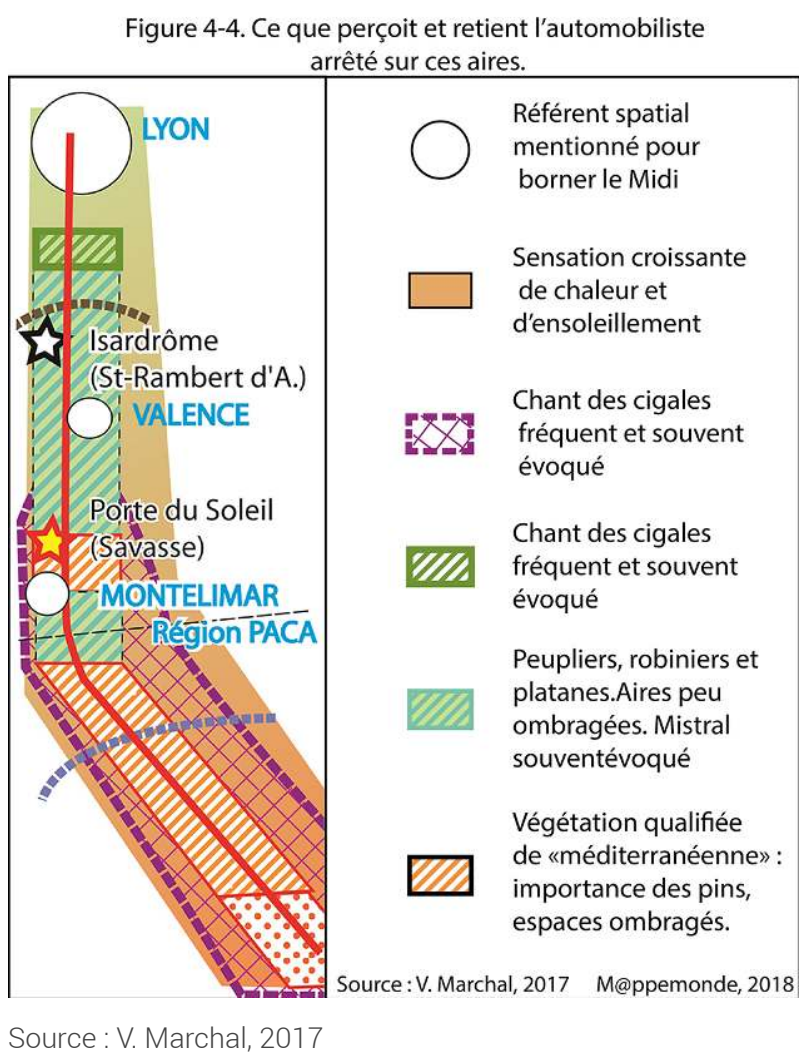

Les grandes aires de service : étapes symboliques, un aménagement total

Avec une fréquentation quasi continue, les aires de service principales ${ }^{10}$, échelonnées tous les $80 \mathrm{~km}$ environ, proposent les prestations les plus complètes (carburants, boutiques, restaurants, parfois hôtels et point détente). Par leurs dimensions, par les annonces anticipées d'une pause reconstituante (signalement $20 \mathrm{~km}$ en amont), ces aires géantes instaurent un rythme régulier sur le parcours et dans le paysage (végétation, services, passerelles). Elles sont aussi le fleuron des sociétés exploitantes, et bénéficient à ce titre de la mise en scène la plus soignée.

Sur le plan végétal, chaque couple d'aires s'organise selon un modèle centre-périphérie : les espèces les plus méridionales investissent les abords les plus fréquentés, proches de la passerelle et des nombreuses ressources (figures 5 à 8), comme s'il s'agissait de rappeler que l'automobiliste circule sur l'autoroute «du soleil», quelle que soit la latitude. Des aires de Beaune à celles de Lançon, la lavande est l'essence la plus plastique, la moins sensible aux gradients latitudinaux, et peut-être la métaphore provençale la plus évocatrice et attendue. Le cyprès s'invite également aux abords des restaurants, mais sous deux modes différents de part et d'autre de la capitale des Gaules : décliné en une variété naine et robuste à Beaune-Tailly et Mâcon Saint-Albain, c'est à Saint-Rambert-d'Albon qu'il prend le port élancé si familier dans les campagnes du Midi. Sur les très méditerranéennes aires de Lançon-de-Provence, même au milieu des pins d'Alep, palmiers et lauriers font figure de végétation d'exception, annonçant l'étroit domaine biogéographique de la côte, encore distante d'au moins $40 \mathrm{~km}$. La métaphore du « décor » proposée par Boutefeu convient pour qualifier ce petit écrin méditerranéen où les limites 
de répartition climacique sont forcées (figure 9) ${ }^{11}$. En revanche, en dehors des points les plus fréquentés (station-essence, boutiques, restaurants), les périphéries des aires portent une végétation en harmonie avec celle de la région, cherchant à éviter toute répétition d'une aire à l'autre, qui serait peu compatible avec l'idée même de transition. Le hêtre clôture les aires de Beaune, celles de Mâcon, en plaine de Saône, voient s'épanouir peupliers hybrides et acacias. Chêne vert et chêne pubescent ménagent sur les haltes de Montélimar des zones de repos ombragées, dans un décor digne des boisements subméditerranéens qui garnissent les versants tout proches (photo 7). À Lançon, le pin d'Alep est chez lui (Lenoble, 1921) et couvre plus de la moitié des espaces réservés au stationnement, à l'image des terres environnantes.

Figure 5. Traitement végétal des deux principales aires de service sur la section Beaune-Lyon concédée à APRR

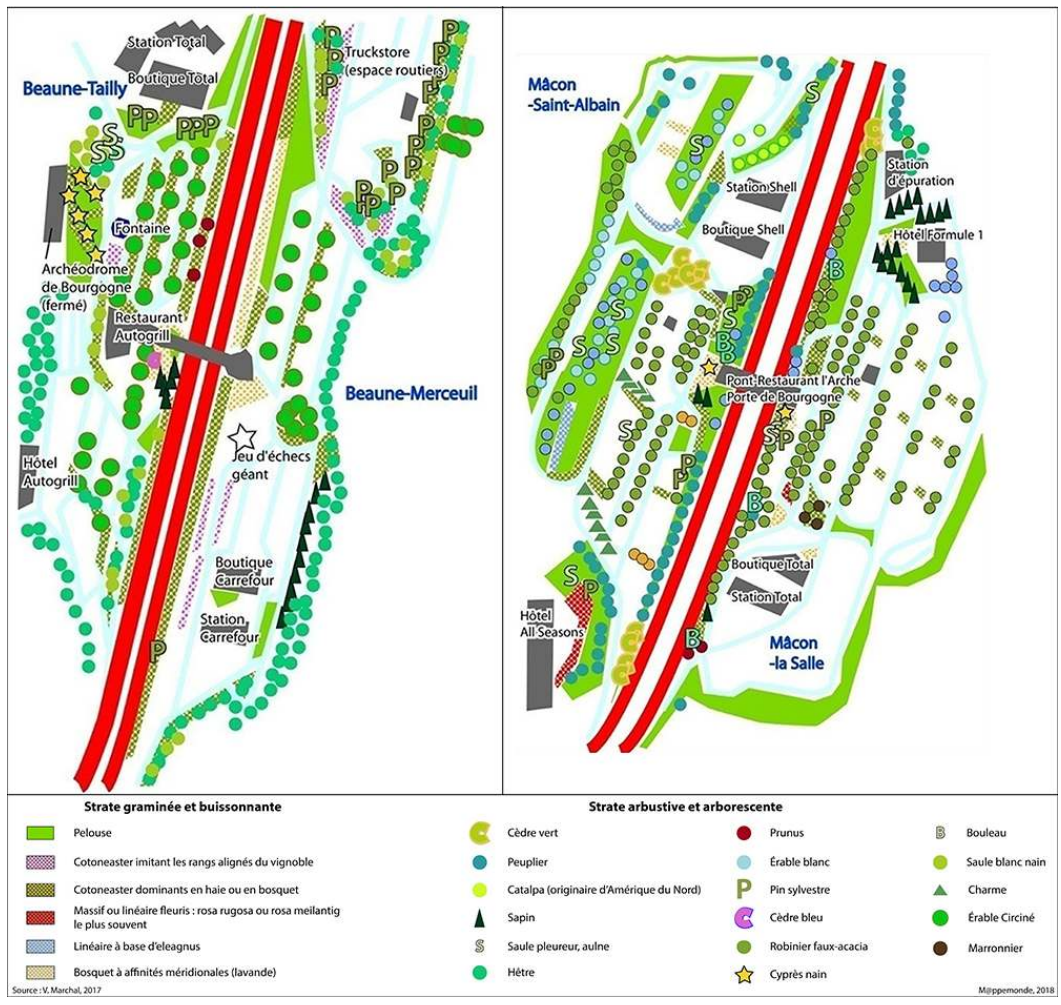

Source : V. Marchal, 2017 
Figure 6. Aménagements végétaux sur les aires de Saint-Rambert d'Albon (ISARDOME)

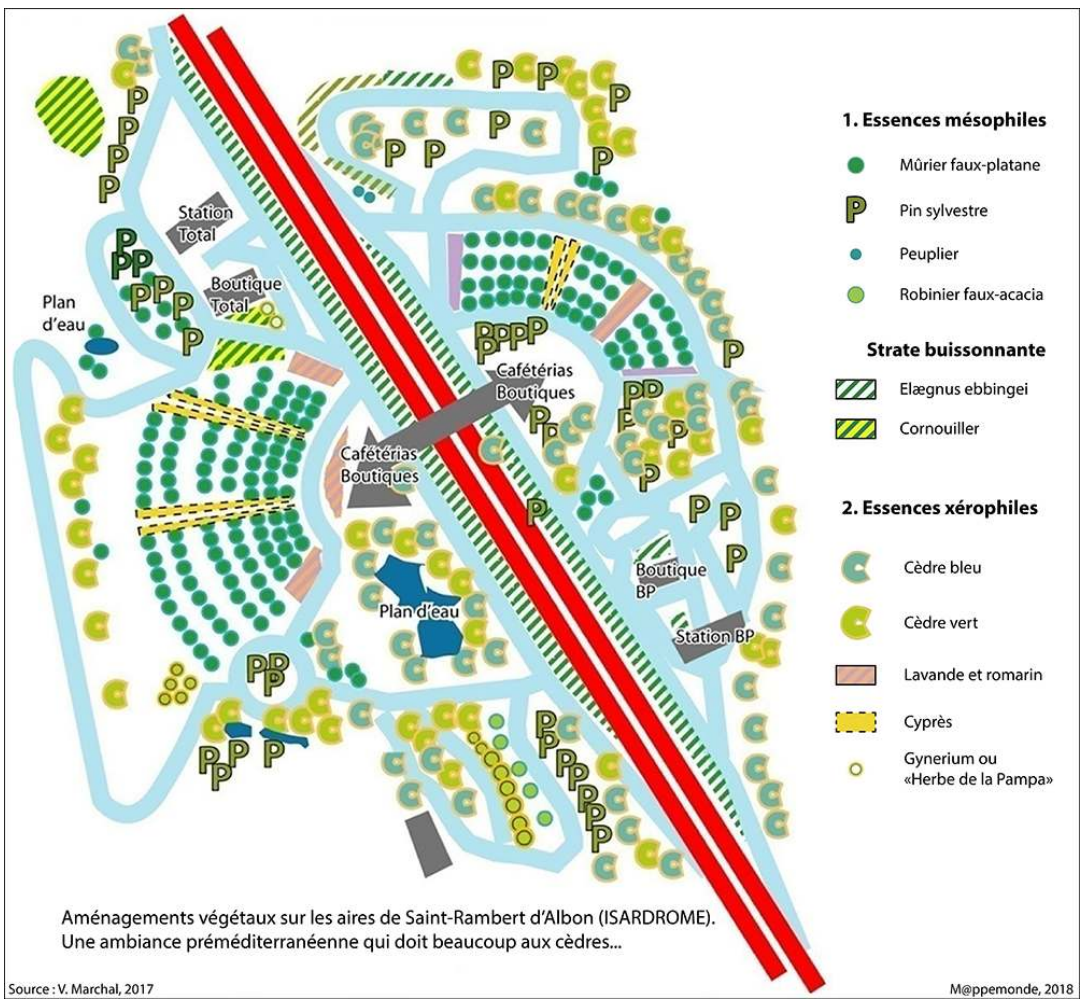

Une ambiance préméditerranéenne qui doit beaucoup aux cèdres...

Source : V. Marchal, 2017

Figure 7. Le traitement végétal des aires de Montélimar

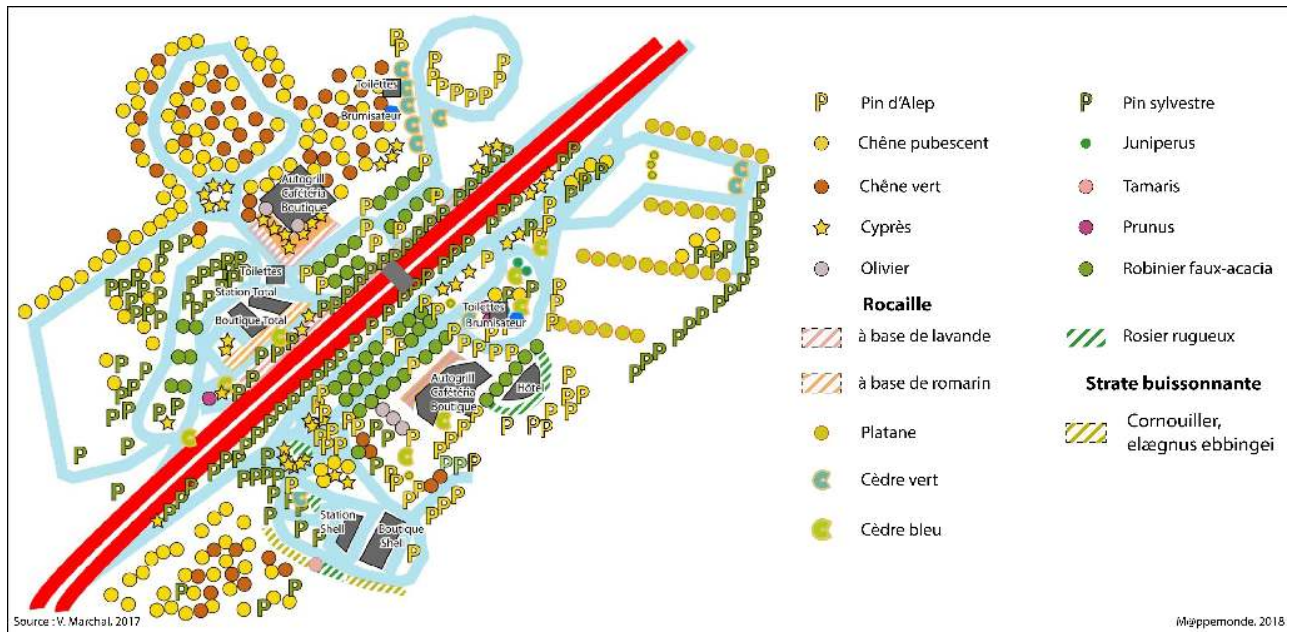

Source : V. Marchal, 2017 
Figure 8. Traitement végétal des aires de Lançon-de-Provence

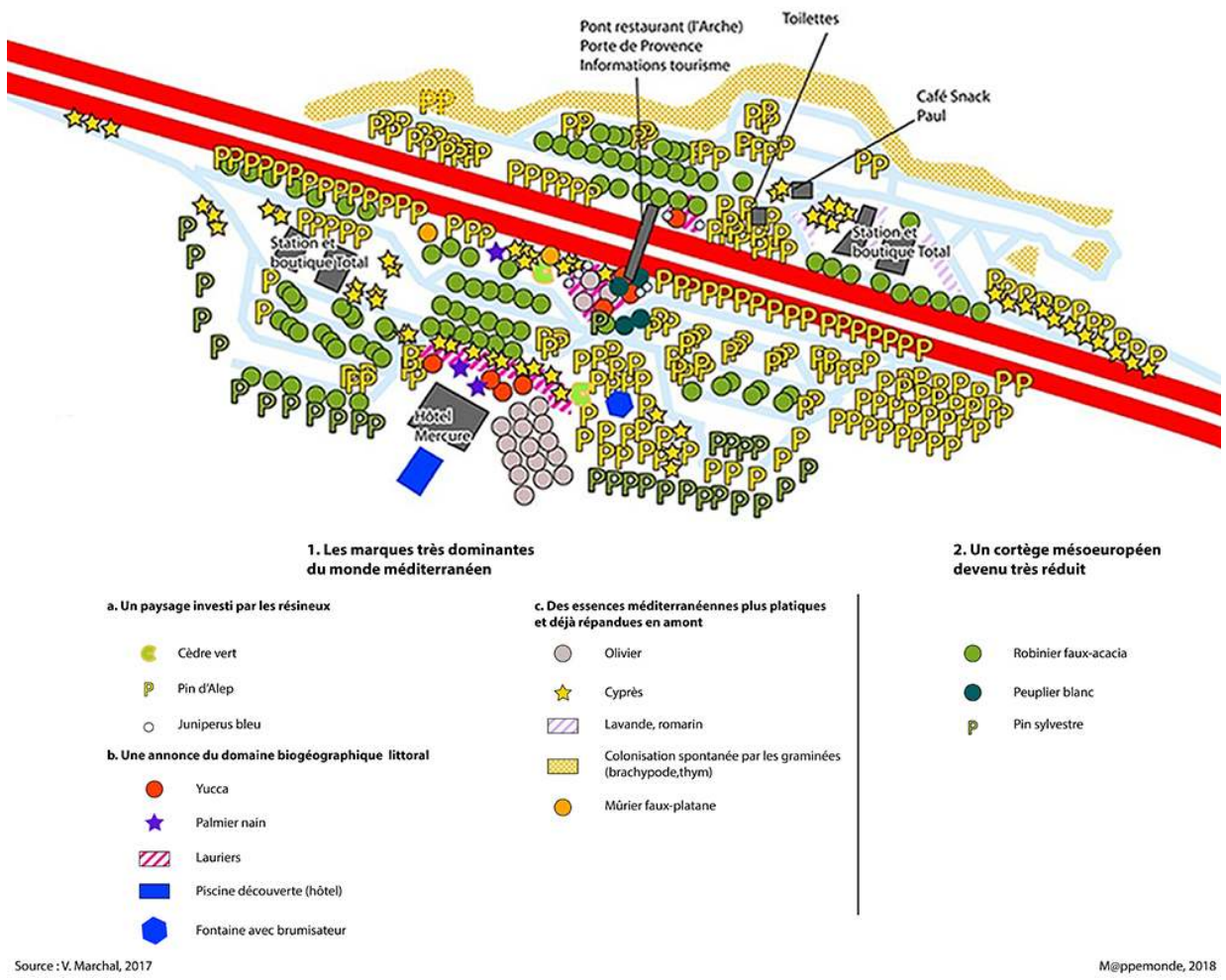

Essai de cartographie

Source : V. Marchal, 2017 
Figure 9. Aires de service et logique de répartition des ambiances végétales

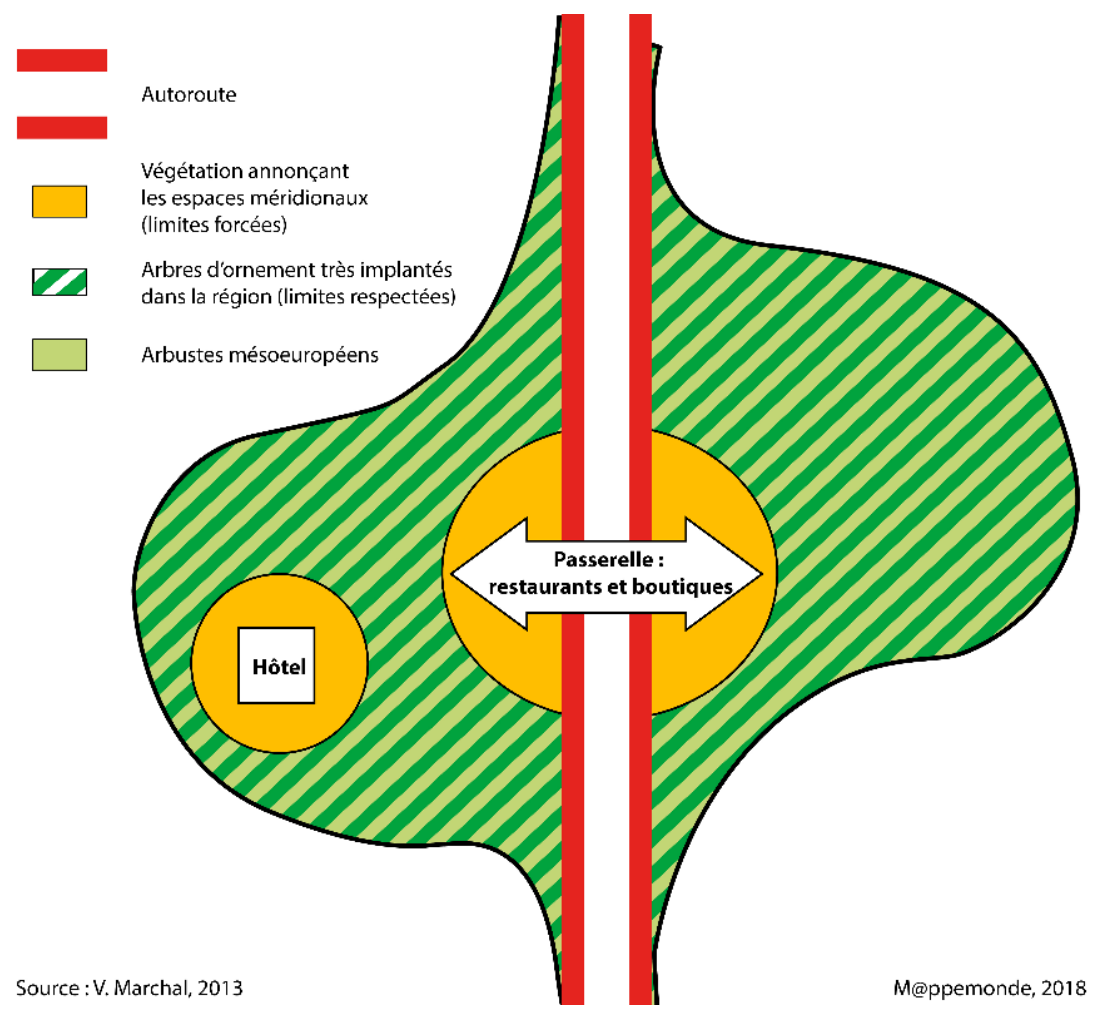

Source : V. Marchal, 2013 
Photo 7. Invitation au repos ou au pique-nique dans une atmosphère digne des sous-bois méditerranéens

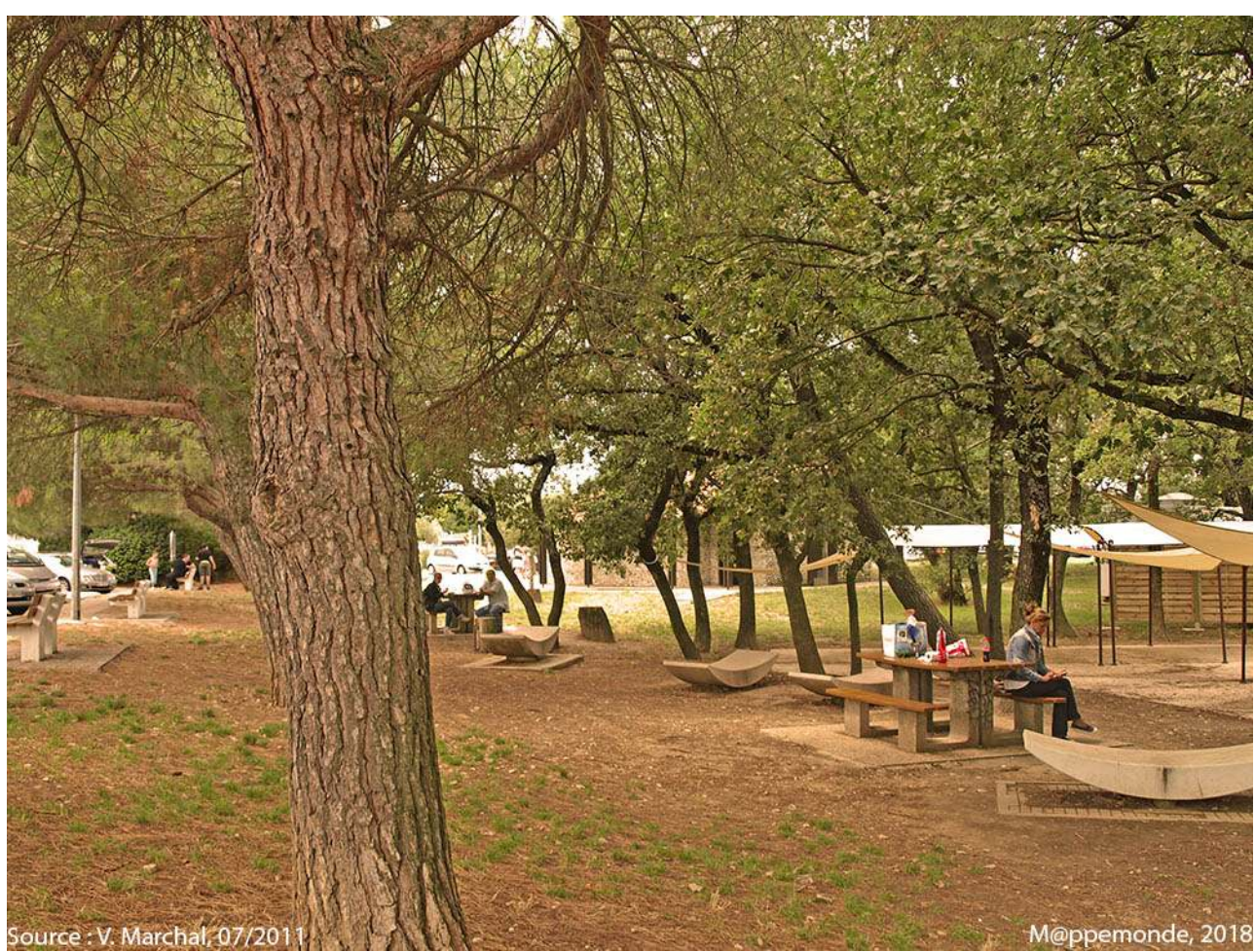

Aire de Montélimar

Cliché V. Marchal, 07/2011

\section{Une mise en tourisme qui efface peu à peu la transition ?}

Si le végétal constitue a priori une trame de fond devant garantir l'immersion du voyageur dans des atmosphères nouvelles, l'architecture des lieux cristallise aussi les marques du changement. Néanmoins, les contraintes d'aménagement propres au milieu autoroutier (bâtiments et stationnement normés, signalétique) imposent fatalement un paysage de la répétition, presque banal, où l'équation entre fonctionnalité et personnalité est difficile à trouver.

\section{Une transition architecturale appelée à s'effacer}

La genèse de l'Autoroute du Soleil s'inscrit dans le contexte du fonctionnalisme et de la standardisation architecturale des 30 Glorieuses. Dans la décennie 1970, pontsrestaurants, stations et boutiques répètent leur esthétique calibrée, incarnant la modernité du milieu autoroutier (figure 10). Cependant, cette modernité triomphante n'était pas incompatible avec le désir d'affirmer une identité locale. Dès l'origine, l'architecture des sanitaires épouse les variations architecturales de la Bourgogne à la mer, en pastichant les variantes locales: les toitures en tuile plate, typiques de la Bourgogne du Nord (photo 8), sont remplacées au sud de Tournus par la tuile-canal mâconnaise, tandis que la pente des toits a perdu $20 \%$. Ce choix respecte exactement la limite entre les deux types de tuiles, qui suit une ligne Tournus-Louhans (Claval, 1978). À partir de Vienne, le style des bâtiments réservés aux sanitaires et aux douches apporte la 
sensation que l'essentiel a déjà eu lieu plus au nord: la tuile-canal règne en effet depuis le Mâconnais, et la toiture à quatre pans, si répandue en moyenne vallée du Rhône, est généralisée jusqu'aux portes de Marseille (photo 9). L'habillage des murs en galets roulés, disposés en "arête de poisson", est répandu dans le nord de la Drôme, mais il se rencontre de manière ponctuelle sur les murets et les petites constructions sur toutes les terrasses alluviales du Rhône et de la Durance.

Figure 10. Les principales tendances d'aménagement des grandes aires de service depuis 1970 : entre identité régionale et fonctionnalisme

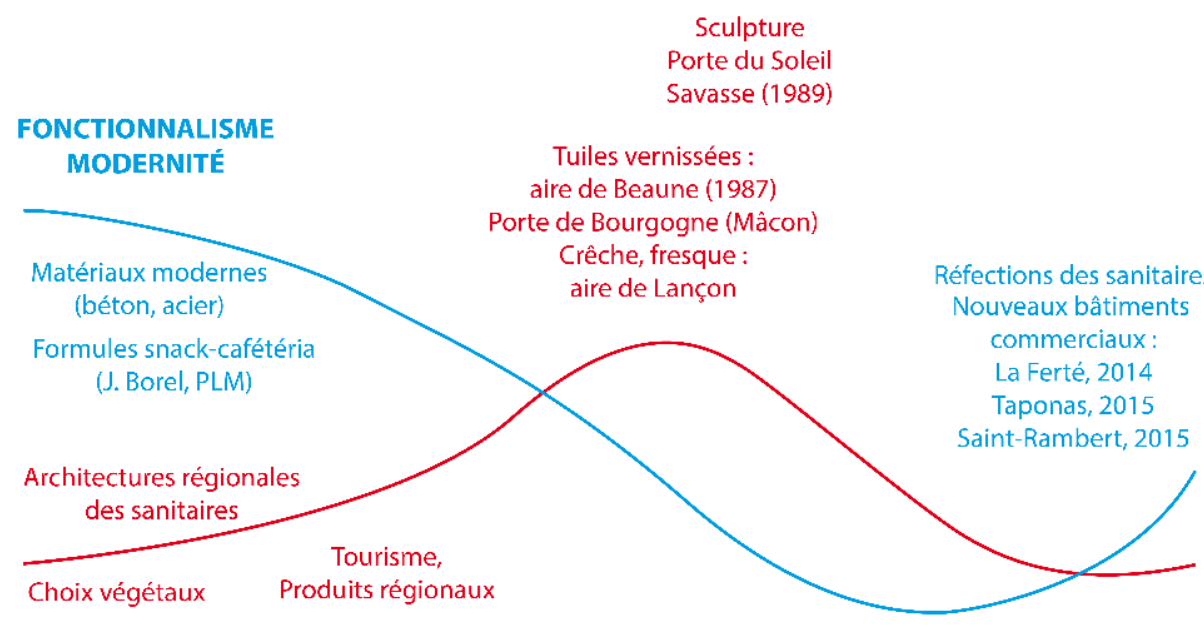

IDENTITÉ RÉGIONALE

1970

1980

1990

2000

2010

Source : V. Marchal, 2017

Mœppemonde, 2017

Source : V. Marchal, 2017 


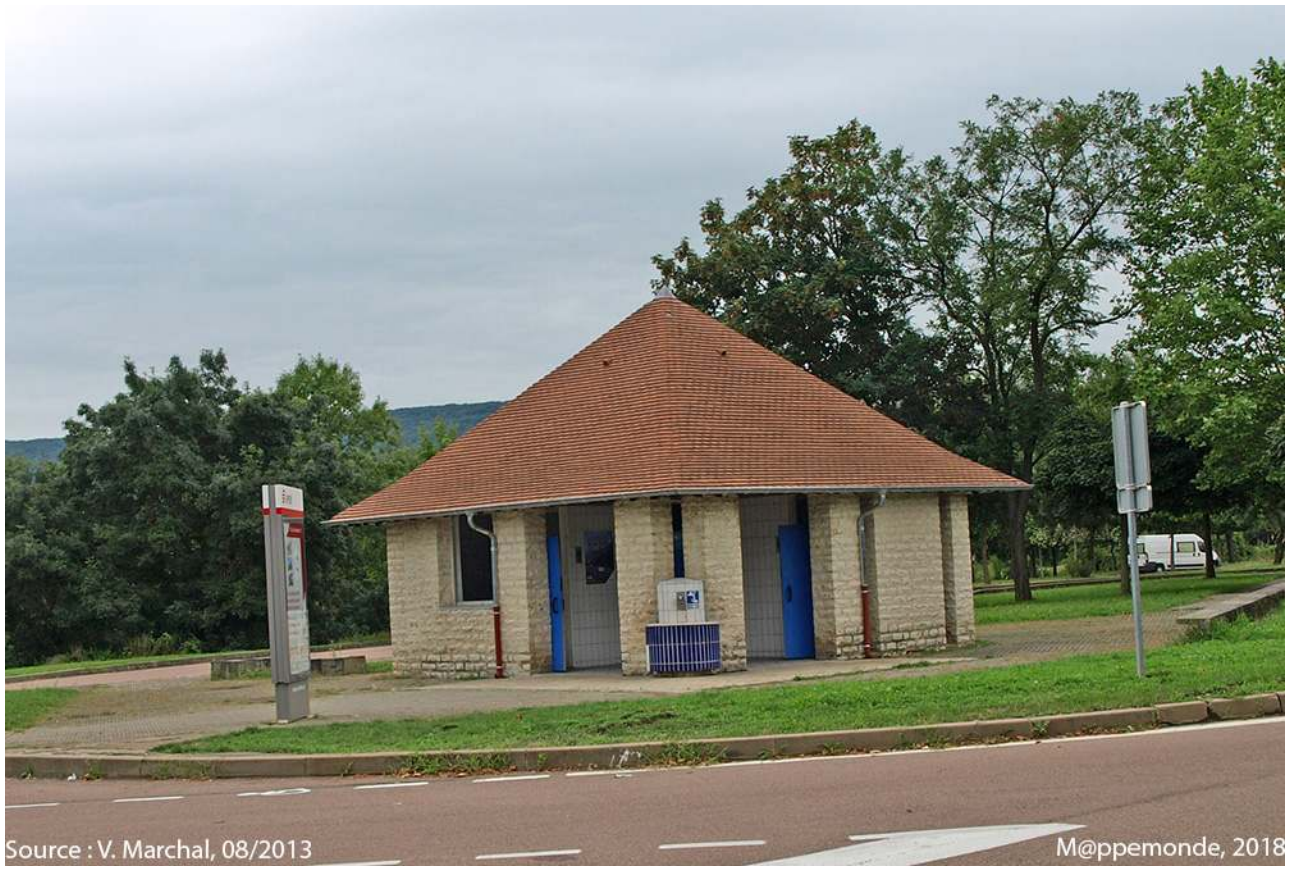

Tuile plate et forte pente sont encore typiques de la Bourgogne du nord.

Cliché V. Marchal, 08/2013

Photo 9. Murs en galets roulés, tuile-canal sur l'aire de Lançon

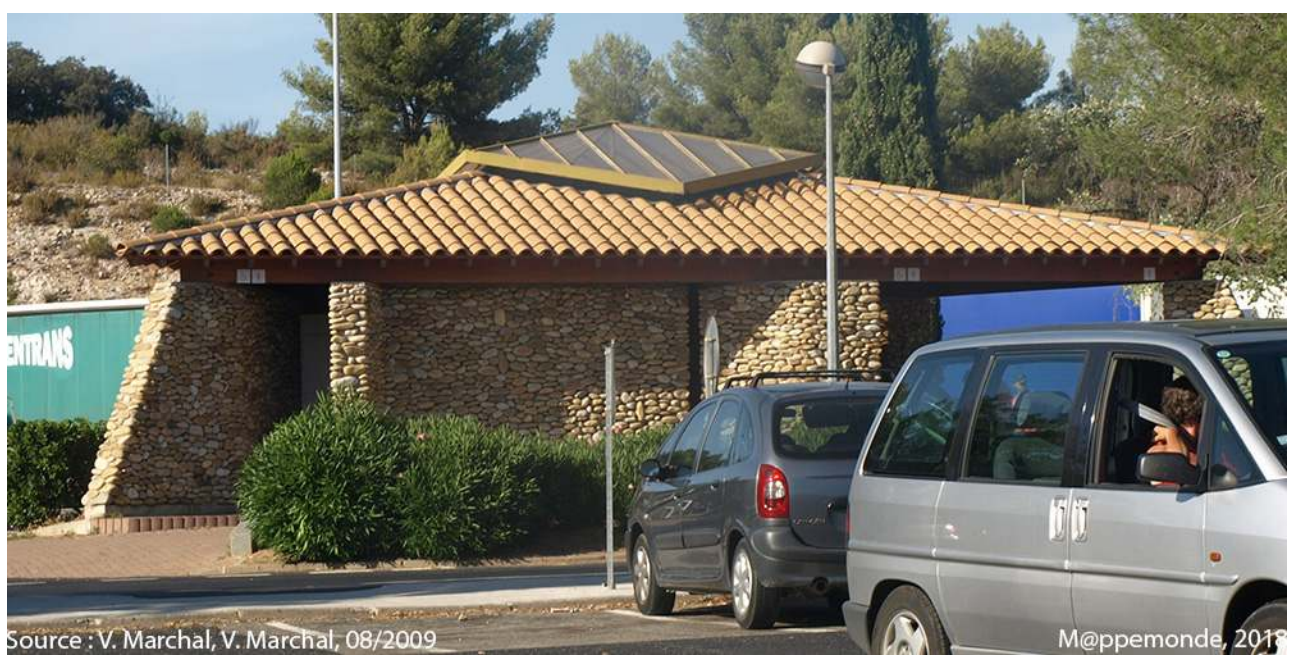

De Lyon à Marseille, l'architecture des sanitaires ne connaît que des variations mineures.

Cliché V. Marchal, 08/2009

Cette volonté de prêter aux aires un cachet régional culmine dans les années 1980, et répond au rejet d'une modernité accusée d'avoir effacé les identités locales, les mémoires de territoire. Le monde autoroutier incarne alors un "anti-paysage », un "non-lieu » (Augé, 1992) qui doit être ré-humanisé12. En 1987, la passerelle des aires de Beaune est habillée d'un revêtement imitant les toitures des célèbres hospices (photo 10), celle de Mâcon est baptisée « Porte de Bourgogne » en 1988, enrichie d'une image de vignoble à 
partir de 1992. À Lançon, au pied du pont-restaurant, une fresque tente d'incarner les images associées aux vacances en Provence (soleil, baignade, oliviers, lavande).

Photo 10. Parure de nuit pour la passerelle des aires de Beaune, avec une reconstitution des célèbres toits polychromés de l'Hôtel-Dieu

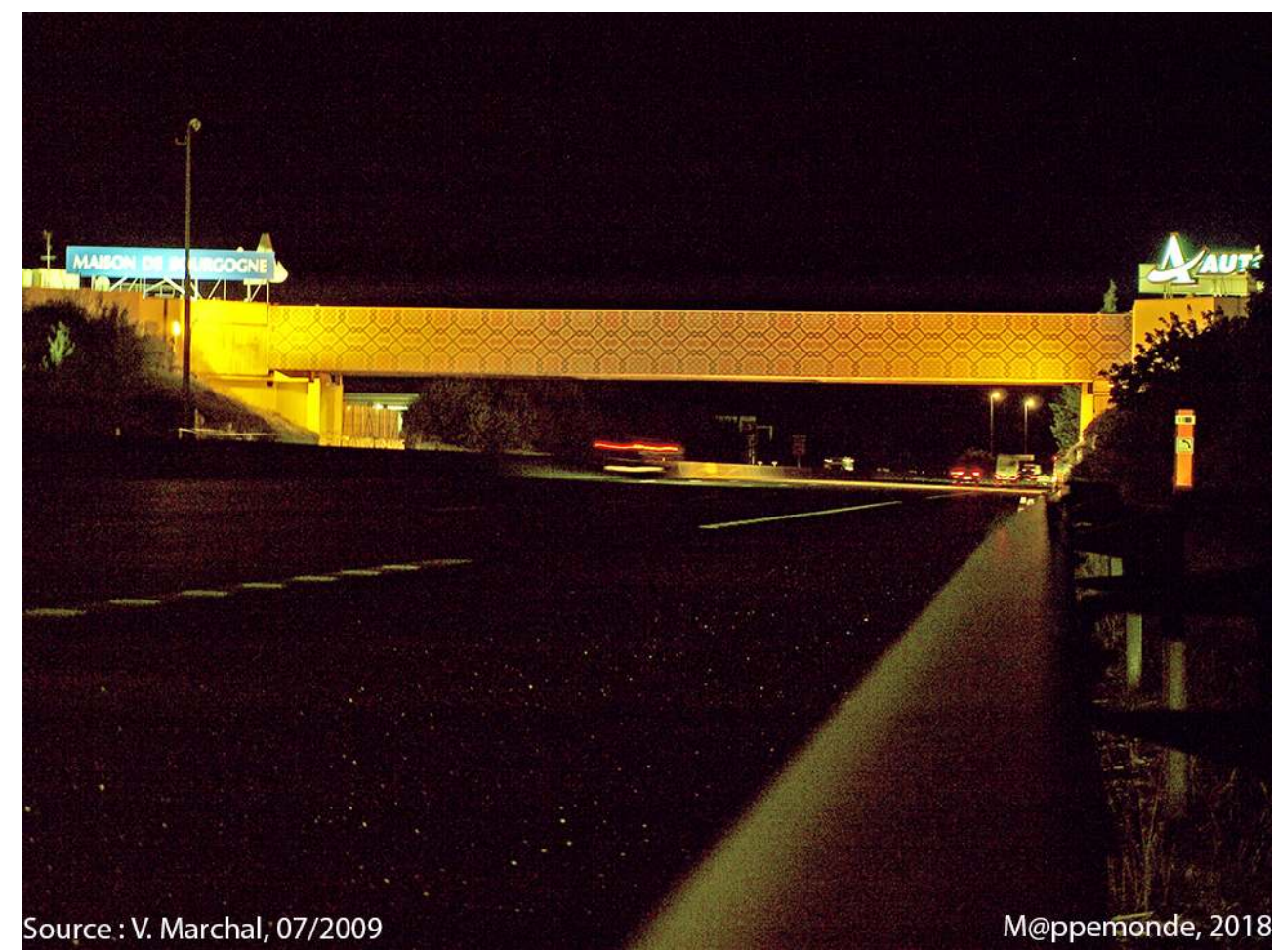

Cliché V. Marchal, 07/2009

Stéréotypées, vieillissant mal, ces initiatives semblent s'effacer à partir des années 2000, où un nouveau désir de modernité gagne les aménagements autoroutiers (figure 10) : remplacement des sanitaires par des constructions plus "design", plus propres et écologiques (mais sans lien explicite avec le style local), préférence affirmée pour une architecture légère, aérienne, laissant entrer les éléments naturels (soleil, vent), signe d'un futurisme assumé et soucieux de l'environnement (aires de la Ferté, de Taponas, de Saint-Rambert-d'Albon) où la référence au contexte régional n'est plus qu'indirecte (espaces protégés du vent, ombragés).

\section{Des portraits sélectifs qui refusent parfois la transition}

Créateur d'ambiances, le monde végétal s'accommode aisément de l'incertitude de la transition; il est propice à suggérer autant d'atmosphères que de nuances. Mais à première vue, l'hésitation entre Nord et Midi se prête moins aux opérations de marketing territorial, surtout s'il s'agit de "vendre " aux estivants de passage un territoire idéel, digne d'une carte postale, sans fausse note ni discordance: ainsi, La Porte du Soleil (sculpture d'Ivan Avoscan, 1989) installée sur l'aire de Savasse, exprime une négation de la transition, faisant du pays de Montélimar une rupture au-delà duquel s'ouvre l'univers méditerranéen. En sens inverse, l'aire de la latitude 45 au nord de Valence exprime davantage une étape du passage progressif entre la France du Midi et celle du Nord. 
31 Le sens à donner aux aires de service peut venir d'une identité régionale puissamment ancrée dans l'histoire, l'économie et la société des terres abordées. Bourgogne et Provence prêtent à trois aires principales sur cinq une raison d'être, revendiquée parfois jusque dans leur dénomination. Charles Trénet le suggère déjà en 1959 lorsqu'il chante la « route des vacances, qui traverse la Bourgogne et la Provence $»^{13}$; l'autoroute n'a fait que reprendre cette attente. Sur moins de 100 kilomètres, deux grandes aires sont dédiées à la Bourgogne, mais elles sont complémentaires, et restituent le sentiment d'une région à plusieurs visages, parcourue par des fractures auxquelles la transition entre le Nord et le Sud de la France n'est pas étrangère (Claval, 1978). Celles de Beaune se consacrent au monde du vignoble de réputation mondiale, si proche de l'autoroute (reconstitution artificielle du vignoble sur l'aire de Beaune-Merceuil). Les haltes de Mâcon s'ouvrent davantage sur la Bourgogne méridionale, moins célèbre, mais les références à la vigne et à l'image d'une région verte compensent ce déficit.

Véritable région-stéréotype, but ultime du voyage pour beaucoup d'usagers de l'autoroute du Soleil, la Provence imprime sa marque sur les aires de Lançon, dernier grand pôle de services avant le littoral : pont baptisé "Porte de Provence ", boutiques consacrées aux produits régionaux, cafétéria proposant des plats méridionaux dans une ambiance provençale (nappes jaunes et bleues...), reconstitution d'une crèche de Noël à l'entrée de l'hôtel.

\section{Les identités intermédiaires : un marketing territorial difficile}

Beaune et Mâcon sont sans conteste bourguignonnes, Lançon revendique son ancrage en Provence jusque dans son nom. Mais pour les haltes situées en terre de contact empruntant des références à plusieurs espaces externes, la mise en marque des territoires peut-elle jouer sur les mêmes registres?

34 Au sud de Lyon, deux grandes aires illustrent le problème: celle de Saint-Rambertd'Albon, à la pointe nord du département de la Drôme (mieux connue sous le nom « Isardrôme ») et de Montélimar. Les deux localités ont en commun leur situation dans la vallée de Rhône, un profil agronomique assez proche (importance des céréales et de l'arboriculture), mais se distinguent sensiblement sur le plan climatique.

Le concept Isardrôme, lancé en 1965 avec l'inauguration de la section Tain-Chanas, avait pour objectif de faire naître une territorialité aux confins de la Drôme, de l'Isère et de l'Ardèche. L'initiative visait à faire connaître des usagers de l'autoroute les espaces du triangle Roussillon - Annonay-Saint-Vallier et à promouvoir les productions locales (fruits, vins). La difficulté était de créer un sentiment d'appartenance, une appropriation d'un espace qui n'avait jamais existé auparavant en tant que tel. En 45 ans d'existence, Isardrôme s'est peu exporté en dehors de l'aire de service qu'elle a fini par résumer à elle seule. En réalité, l'identité mise en scène sur la station de l'A7 est très déséquilibrée. Isardrôme reste d'abord une aire consacrée à la Drôme et au Midi approchant : les ravioles de Royans, les vins de Crozes-Hermitage trouvent leur place, mais ils doivent déjà s'affirmer face au nougat de Montélimar, à la lavande et au vignoble de Tricastin, qui ont droit à un présentoir intitulé « aux portes du soleil ». L'Ardèche est peu présente dans la promotion territoriale, si ce n'est à travers quelques produits locaux comme les marrons glacés, la crème de châtaignes ou le vignoble de Saint-Joseph. L'Isère, pourtant voisine de 3 kilomètres, est totalement absente de la vente et de la publicisation touristique. 

référents territoriaux ne sont pas les mêmes. Cette fois, la halte est uniquement dédiée à la cité du nougat, et celui-ci tient une place de premier choix dans les cinq boutiques que comptent les deux aires ( 36 présentoirs, 6 étalages, 19 fabricants différents dans une seule boutique !). Pour les maisons de vente et les producteurs locaux, la station autoroutière est devenue le point de contact privilégié avec la clientèle de passage. Le symbole de cette conquête de l'autoroute par le tissu local est le GIE Internougat, né de la volonté des producteurs d'obtenir des points de vente sur l'A7 afin de retrouver leur clientèle ayant délaissé la RN7. Aujourd'hui, l'association tient à maintenir la place des productions locales sur une aire dont elle est une vitrine connue. La Provence, périphérie proche et envahissante, y occupe déjà une place de choix, puisque $38 \%$ des articles y sont associés, des calissons aux sacs à main avec imprimé «champ de lavande ", en passant par les cigales en céramique.

Les aires de Saint-Rambert-d'Albon et de Montélimar ont en commun une promotion à fort caractère économique, centrée sur la vente des produits locaux (plus que régionaux). Les deux stations disposent d'un point de vente de fruits (pêches, brugnons, abricots, melons) en période estivale, exutoire privilégié pour les producteurs ayant partiellement perdu la clientèle de la RN7. L'absence de bureau de tourisme est une autre singularité : terres de transition, mais surtout espaces de l'entre-deux, difficiles à saisir dans leur originalité, les identités intermédiaires de la vallée du Rhône ne sont ni des régionspaysages comme la Provence, ni des régions-terroirs suffisamment affirmées comme la Bourgogne (figure 11).

Figure 11. Aires, aménagements autoroutiers et mise en évidence des transitions : essai de modélisation

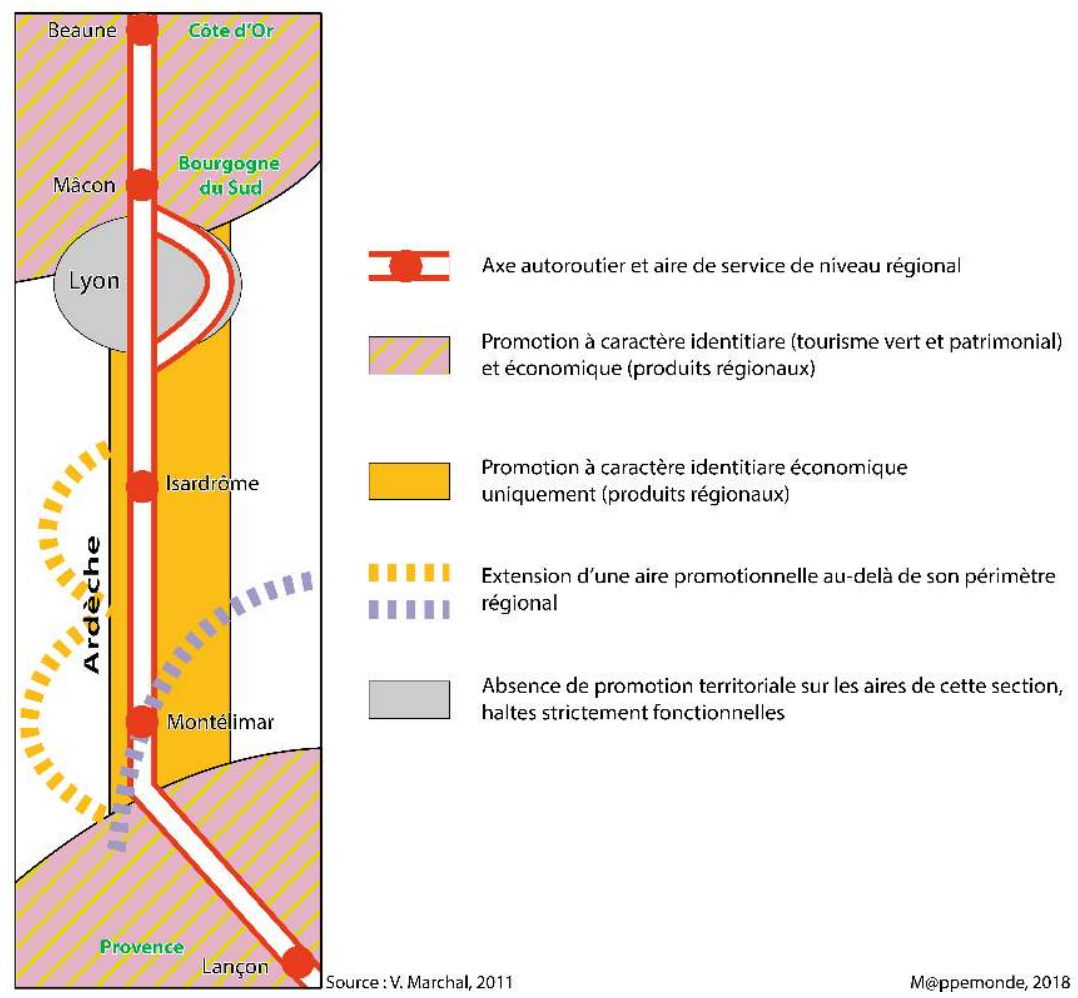

Source : V. Marchal, 2011 


\section{Conclusion}

Comme l'intrigue d'une pièce de théâtre, le passage du Nord au Midi est un horizon d'attente puissant pour les usagers de l'axe le plus fréquenté de France. Les témoignages recueillis en première partie ont montré à quel point il était difficile de dissocier la sensation de dépaysement (en grande partie liée à l'imaginaire générique des vacances) et la quête du Midi méditerranéen (transition, appuyée sur des références et des expériences géographiques concrètes, immédiates). Si une majorité de vacanciers en partance sont polarisés par une approche utilitaire-sécuritaire de l'infrastructure parfois exclusive (liée aux conditions de circulation, à la consommation des services des aires), l'immersion progressive dans un univers méditerranéen confondu avec celui des vacances devient la principale clé de lecture du paysage des aires, à partir de la vallée du Rhône. Aménagés par les sociétés concessionnaires dans un esprit de diversité paysagère symbolisant une intégration réussie de l'infrastructure dans son environnement, les décors végétaux retiennent surtout l'attention des usagers par l'expérience sensorielle qu'ils génèrent (ombre-soleil, effluves, chant des cigales) que par la diversité de leur flore (oliviers, cyprès, pins sont les seules essences véritablement citées pour leur rôle métonymique). Les évolutions récentes des aménagements des aires vont dans le sens d'une transition bioclimatique renforcée par une végétation à maturité, tandis que la promotion identitaire des espaces traversés est devenue aujourd'hui moins fondamentale (en architecture, abandon des pastiches régionaux véhiculant une image à la fois stéréotypée et banale, qui finit par renvoyer l'intention identitaire à «sa propre inconsistance »: Éco, 1985), au profit d'une nouvelle esthétique articulant futurisme et écologie dans une nouvelle urbanité.

\section{BIBLIOGRAPHIE}

AUTOROUTES DU SUD DE LA FRANCE (1984). Rapport d'activité 1984. Avignon, 57 p.

AUGÉ M. (1992). Non-lieux. Introduction à une anthropologie de la surmodernité. Paris : Le Seuil, 158 p. ISBN 9782021290622

BERQUE A., LASSUS B., ROGER A. et al. (1994). Cinq propositions pour une théorie du paysage. Paris : Champ Vallon, coll. « Pays - paysages », $128 \mathrm{p}$.

BIGANDO E. (2006). « La synecdoque paysagère, une notion pour comprendre les représentations des paysages viticoles bordelais et bourguignon ». Sud-Ouest européen, n²1, p. 83-93.

BOUTEFEU B. (2007). La forêt comme un théâtre ou les conditions d'une mise en scène réussie. Thèse de doctorat sous la direction de Arnould P., Lyon, École normale supérieure lettres et sciences humaines/Office national des forêts, $519 \mathrm{p}$.

ClAVAl P. (1978). Atlas et géographie de Haute-Bourgogne et Franche-Comté. Paris : Flammarion, coll. « Portrait de la France moderne », 328 p. 
CORTAZAR J., DUNLOP C. (1983). Les autonautes de la cosmoroute, ou un voyage intemporel Paris-Marseille. Paris : Gallimard, 280 p.

DONADIEU P., PERIGORD M. (2005). Clés pour le paysage. Paris : Ophrys, coll. « Géophrys », 368 p. DUBESSET P. (1972). « Choix agricoles et caractères du climat dans la région du Rhône moyen ». Revue de géographie de Lyon, vol. 47, n² 2, p. 297-326.

DUPUY G. (1995). Les territoires de l'automobile. Paris : Anthropos, coll. « Villes », 216 p. ISBN 9782717828801 ÉCO U. (1985). La guerre du faux. Paris : Grasset, coll. « Les cahiers rouges », 420 p. GARRAUD L. (2005). Flore de la Drôme. Atlas écologique et floristique. Gap : Conservatoire botanique national alpin de Gap-Charance, 925 p. ISBN 2-9511864-1-X

GAY J.-C. (1994). « La mise en décor d'un paysage. La signalisation autoroutière d'animation de la société ESCOTA en Provence-Alpes-Côte d'Azur ». L'espace géographique, vol. 23, n² 2, p. 175-185.

HAGERSTRAND T. (1975). “Space, time and human conditions”. In A. KARLQVIST et al., Dynamic allocation of urban space, Lexington: Saxon House Lexington Book, p. 3-14.

IFOP, ASFA (2001). Français, votre perception de l'autoroute en 2001. Paris, Baromètre image de l'autoroute, $16 \mathrm{p}$.

JARDIN A., FLEURY P. (1973). La révolution de l'autoroute. L'autoroute et la vie quotidienne des Français. Paris : Fayard, coll. « Le monde sans frontières », 256 p.

KNAFOU R., EQUIPE MIT (2008). Tourismes 1. Lieux communs. Paris : Belin, 320 p. ISBN

978-2-7011-4892-2

LE CCEUR C. (DIR.), AMAT J-P., DORIze L., GAUTHIER E. (2002). Éléments de géographie physique. Cours. Documents. Travaux dirigés. Paris : Bréal, coll. « Grand Amphi Géographie », 448 p.

LENOBLE F. (1921). Les limites de végétation de quelques espèces méditerranéennes dans le bassin moyen du Rhône et les Préalpes sud-occidentales. Revue de géographie alpine, t. 9, n 3, p. 457-470.

MARCHAL V. (2013). Les transitions paysagères sur l'Autoroute du Soleil entre Beaune et Marseille. Thèse de doctorat, Nancy, Université de Lorraine, $516 \mathrm{p}$.

MERRIMAN P. (2007). Driving Spaces. A Cultural-Historical Geography of England's M1 Motorway. Oxford: WileyBlackwell, 320 p. ISBN 978-1-405-13072-1

PAULET J.-P. (2002). Les représentations mentales en géographie. Paris : Anthropos, 152 p. ISBN

2-7178-4507-0

ROGER A. (1997). Court traité du paysage. Paris : NRF Gallimard, coll. « Bibliothèque des sciences humaines », 210 p. ISBN 9782070749386

ROLLAND-MAY C. (1987). « La théorie des ensembles flous et son intérêt en géographie ». L'espace géographique, vol. 16-1, p. 42-50.

TOURNIER M. (2008). L'aire du muguet. Paris : Gallimard, 97 p. ISBN 9782070356409

\section{NOTES}

1. Résultat des enquêtes réalisées par l'auteur à partir de 1000 témoignages recueillis sur l'Aire de Lançon-de-Provence, entre 2005 et 2010, lors des grandes migrations estivales. Une des questions posées était: "quelle est la raison principale de votre arrêt sur cette aire?». 
866 personnes interrogées citaient des considérations très pratiques, liées aux services proposés par l'aire (sanitaires, restauration, boutiques), et à la fatigue cumulée.

2. Enquête personnelle, réalisée dans le cadre de ma thèse de doctorat, Les transitions paysagères sur l'Autoroute du Soleil entre Beaune et Marseille, soutenue en novembre 2013.

3. Dans son Court traité du paysage, l'auteur analyse le dépaysement comme une sensation d'évasion hors des territoires du quotidien, hors de l'approche utilitaire de nos espaces de proximité. Distinguant "pays» (territoires de vie, lus et pratiqués de façon fonctionnelle) et "paysage» (lecture sensible, volontaire et esthétisée d'une portion d'espace choisie), A. Roger substitue au «dépaysement» le néologisme «dépaysagement», soulignant l'importance du rapport esthétique à l'espace.

4. Carte-guide des Autoroutes Paris-Rhin-Rhône, 1995.

5. L'analyse sémantique de la notion de Midi révèle que celle-ci s'apparente bien plus à une catégorie d'espace qu'à un domaine géographique précisément borné. Elle est une facette d'un système de représentation antinomique Nord/Midi, l'un étant défini comme le négatif de l'autre. Voir V. Marchal, 2013, p. 32-36.

6. Dans son essai intitulé Space, time and human conditions. Dynamic allocation of urban space (1975), T. Hägerstrand définit l'espace des mobilités quotidiennes comme un archipel de pôles autour desquels se forge une connaissance fine de l'espace, fruits d'une pratique utilitaire, quotidienne et identitaire des lieux.

7. Le guide de l'autoroute, 1998, p. 78.

8. Surnommée "l'yeuse ", cette espèce au port arbustif et aux feuilles luisantes, légèrement épineuses, est adaptée à la sécheresse méditerranéenne. Elle est une composante fondamentale du maquis.

9. R. Knafou et M. Stock, 2008, Tourismes 1. Lieux communs, p. 8.

10. Le vocabulaire autoroutier distingue aires de service secondaires et principales. Les dernières se distinguent par la taille, le niveau de services (souvent un hôtel) et la présence d'une passerelle.

11. Les piles massives du pont qui enjambe l'autoroute, ainsi que la hauteur des constructions permettent sans doute un effet d'abri qui épargne ces espèces fragiles des assauts du Mistral, des appels d'air incessants des véhicules et des bouffées d'air froid, en particulier hors saison estivale.

12. Les panneaux marron, dits "d'animation », visant à faire découvrir des curiosités proches de l'autoroute, se généralisent à cette période. Sur ce point, voir l'article de J.-C. Gay consacré à l'autoroute A8.

13. Ch. Trénet, voir les paroles de la chanson Nationale 7, 1959.

\section{RÉSUMÉS}

Semblables à des arrêts sur image au cours d'un film, les aires d'autoroute sont des objets géographiques singuliers : si les commodités fonctionnelles ont souvent dicté leur raison d'être, elles sont aussi l'occasion pour le voyageur de nouer un contact éphémère avec les terres traversées. De Beaune à Marseille, la transition entre le nord et le midi de la France sert-elle de fil conducteur aux actions d'aménagement des aires? Pour les usagers de l'Autoroute du Soleil, 
l'approche du monde méditerranéen est un horizon d'attente fort, que le marketing territorial des aires doit aussi concilier avec la promotion des identités locales.

Rest areas resemble freeze-frames of a film and make original subjects of study. Their functions are dictated by their purpose but they also offer travelers a brief glimpse of the surrounding regions. From Beaune to Marseille, could the transition from the north to the south of France be the thread that links the landscaping of this set of rest areas? For many on the Autoroute du Soleil, the Mediterranean is a sharply anticipated destination. The marketing and development of tourism must balance the need to offer previews of the south and their desire to promote local identities.

Como fotogramas de una película cinematográfica, las áreas de servicio de las autopistas son objetos geográficos singulares. Aunque su finalidad es atender al viajero, también sirven para el contacto de este con el espacio que recorrre. En el trayecto de Beaune a Marsella, ¿podría valorarse a través de ellas la transición y cambios en la ordenación territorial entre el norte y el mediodía francés? Para algunos usuarios estas áreas de la Autopista del Sol les evocan unas expectativas sobre el mundo mediterráneo que se potencian por el maquetin y la promoción de la identidad local de esos espacios.

\section{INDEX}

Mots-clés : autoroute, identité, paysage, publicisation, transition

Palabras claves : autopista, paisaje, transición, publicitar, identidad

Keywords : highways, landscape, transition, advertising, identity 